\title{
Evaluation of Methods for In-Situ Calibration of Field-Deployable Microphone Phased Arrays
}

\author{
William M. Humphreys, Jr., David P. Lockard`, Mehdi R. Khorrami ${ }^{\ddagger}$, \\ William G. Culliton ${ }^{\S}$, Robert G. McSwain \\ NASA Langley Research Center, Hampton, Virginia
}

\begin{abstract}
Current field-deployable microphone phased arrays for aeroacoustic flight testing require the placement of hundreds of individual sensors over a large area. Depending on the duration of the test campaign, the microphones may be required to stay deployed at the testing site for weeks or even months. This presents a challenge in regards to tracking the response (i.e., sensitivity) of the individual sensors as a function of time in order to evaluate the health of the array. To address this challenge, two different methods for in-situ tracking of microphone responses are described. The first relies on the use of an aerial sound source attached as a payload on a hovering small Unmanned Aerial System (sUAS) vehicle. The second relies on the use of individually excited ground-based sound sources strategically placed throughout the array pattern. Testing of the two methods was performed in microphone array deployments conducted at Fort A.P. Hill in 2015 and at Edwards Air Force Base in 2016. The results indicate that the drift in individual sensor responses can be tracked reasonably well using both methods. Thus, in-situ response tracking methods are useful as a diagnostic tool for monitoring the health of a phased array during long duration deployments.
\end{abstract}

\section{Introduction}

$\mathrm{T}$

he NASA Flight Demonstrations and Capabilities (FDC) Project promotes focused flight experiments to validate critical technologies, including noise reduction concepts. These flight experiments require the use of measurement diagnostics, both aircraft- and ground-based, in order to quantitatively evaluate the benefit of specific concepts. In the realm of noise reduction characterization, one of the primary tools for such quantitative measurements is the microphone phased array. NASA Langley Research Center (LaRC) has a long history of successfully utilizing microphone arrays in both ground test facilities as well as in flight test campaigns. ${ }^{1-2}$

In 2006 a 167-microphone array was deployed at the NASA Wallops Flight Facility (WFF) to conduct an extensive series of baseline airframe noise measurements on two Gulfstream aircraft. ${ }^{3}$ Figure 1 shows an aerial view of the array deployed on the overrun area of WFF Runway 4 for the campaign. The microphones were low-cost, commodity electret units placed on the runway surface in a central mounting plate and on individual ground plates. While useful measurements were obtained during the 2006 campaign, there were a number of issues with the operational performance of the array that limited its

\footnotetext{
*Aerospace Engineer, Advanced Measurements and Data Systems Branch, Associate Fellow AIAA

$\dagger$ Aerospace Engineer, Computational Aerosciences Branch, Senior Member AIAA

$\$$ Aerospace Engineer, Computational Aerosciences Branch, Associate Fellow AIAA

§Electronics Technician, Advanced Measurements and Data Systems Branch

^Aerospace Engineer, Aeronautics Systems Engineering Branch
}

1 of 22

American Institute of Aeronautics and Astronautics 
ultimate utility. In particular, the microphones were not hardened for use in an outdoor environment. As a result of moisture contamination, the sensitivity of the sensors drifted significantly during the test campaign, making accurate quantitative measurements of noise levels difficult. Furthermore, there was no effective method for calibrating the entire array of microphones in-situ.

In order to address the issues discovered during the 2006 deployment, a completely new array design was developed, suitable for long-duration outdoor deployments where airframe and propulsive noise measurements are desired for a range of aircraft types and scales. The new array ${ }^{4}$ incorporates up to 185 hardened, weather-resistant commercial off-the-shelf sensors (Figure 2) modified

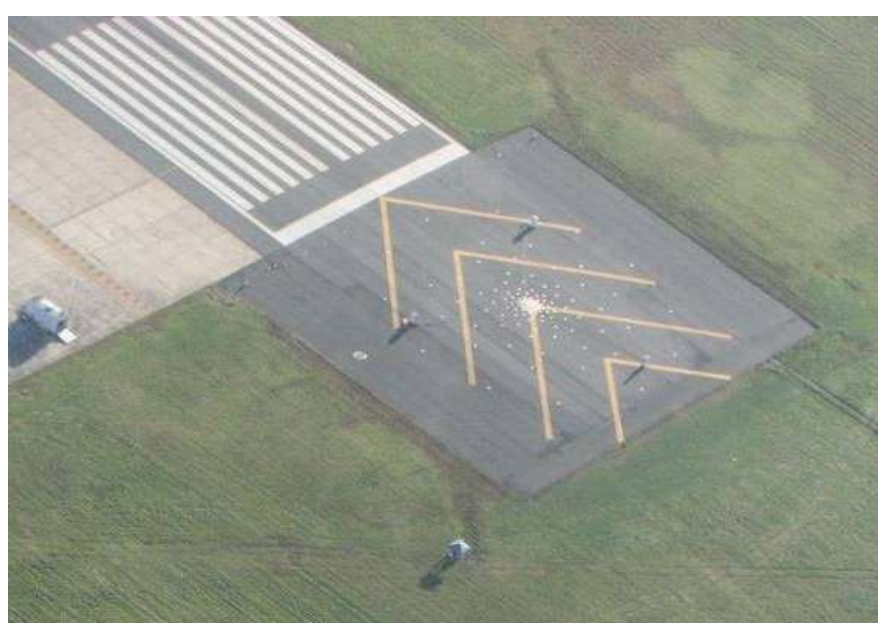

Figure 1. 167-microphone array at NASA WFF in 2006. Dots on overrun area are microphone ground plates. Data acquisition system cabinets are visible around the perimeter of the array. to use a custom 4-mA current loop circuit with temperature compensation to power the sensor over extended cable lengths with minimal degradation of the signal to noise ratio and frequency response. The design of the sensors is described in detail in Ref 4.

The first deployment of the new phased array system occurred at Finnegan Airfield at Fort A.P. Hill in late August of 2015, where the array was utilized to measure the noise from a number of sUAS (small Unmanned Aerial System) vehicles. A starburst microphone pattern 79 feet wide and 93.7 feet long was positioned at the east end of the runway with the edge of the array 35 feet from the runway threshold. An aerial photo of the deployment is shown in Figure 3. The A.P. Hill deployment was followed in August 2016 with a deployment at Edwards Air Force Base (AFB) in California where a 250-foot diameter spiral pattern of microphones was placed near the threshold of Runway 18L and used for flyover measurements of flap and undercarriage noise for two different NASA Gulfstream (G-III) aircraft. An aerial photo of this deployment is shown in Figure 4.

A critical aspect of the array operations at both A.P. Hill and Edwards AFB was the inclusion of calibration methods whereby daily in-situ array sensor response (i.e.,

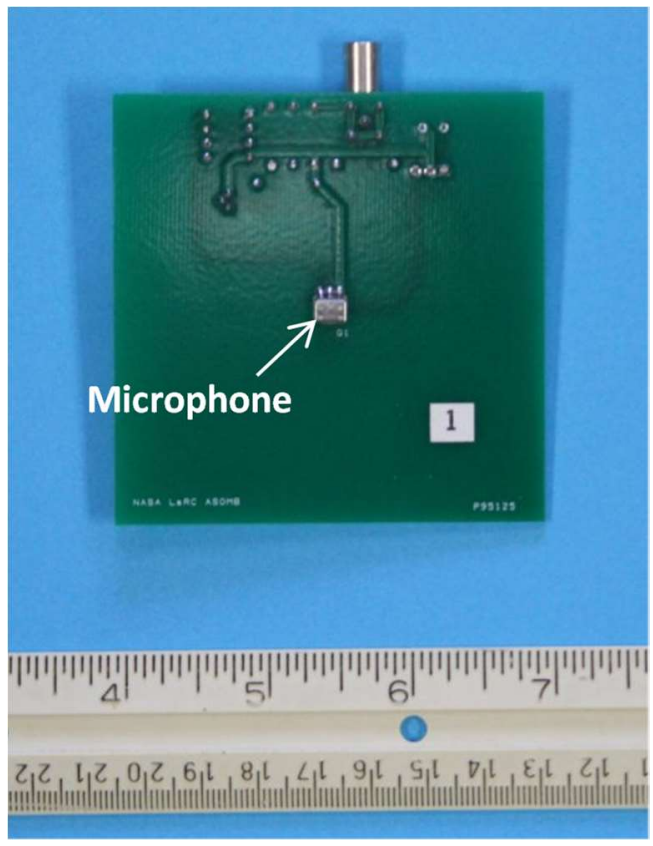

Figure 2. Hardened array microphone mounted on printed circuit board. sensitivity) tracking and channel performance data could be obtained for the duration of the deployment. This is a non-trivial task, given the constraints imposed by the ambient environment (i.e., weather conditions, background noise) as well as the large size of the array aperture. In general, it is not practical to perform a daily calibration of each individual microphone in the array. Therefore, global methods for tracking the response of all of the array elements must be employed. This paper describes two particular methods that were attempted during the A.P. Hill and Edwards AFB deployments: 
1. A unique in-situ calibration method using a hovering aerial sound source.

2. A multiple ground-source calibration method.

Both of these methods are described in Section II. Section III describes the strategy employed to track microphone responses using the two methods with corrections that are performed on the data. Section IV concludes with a comparison of results obtained using the two methods during the deployments.

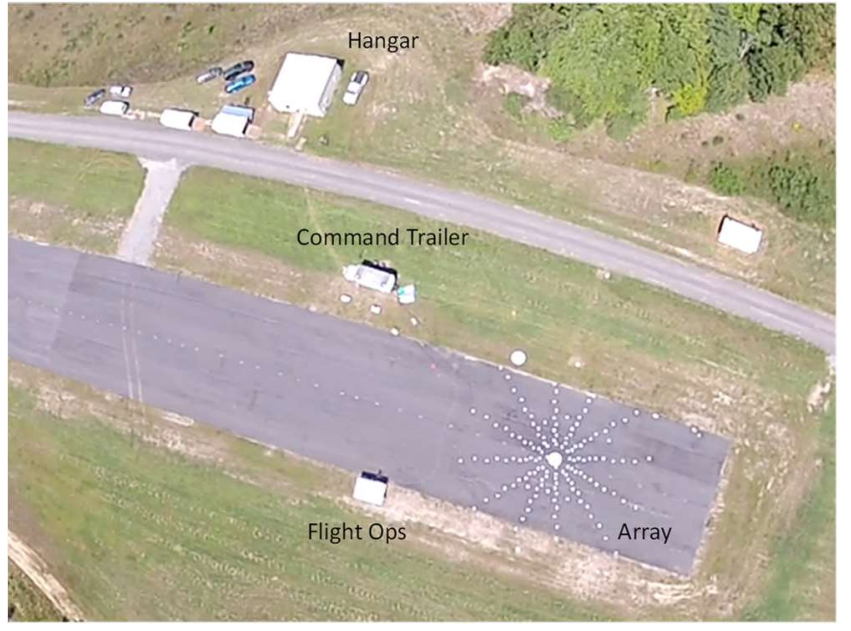

Figure 3. Aerial view of array deployment at Finnegan Airfield, Fort A.P. Hill.

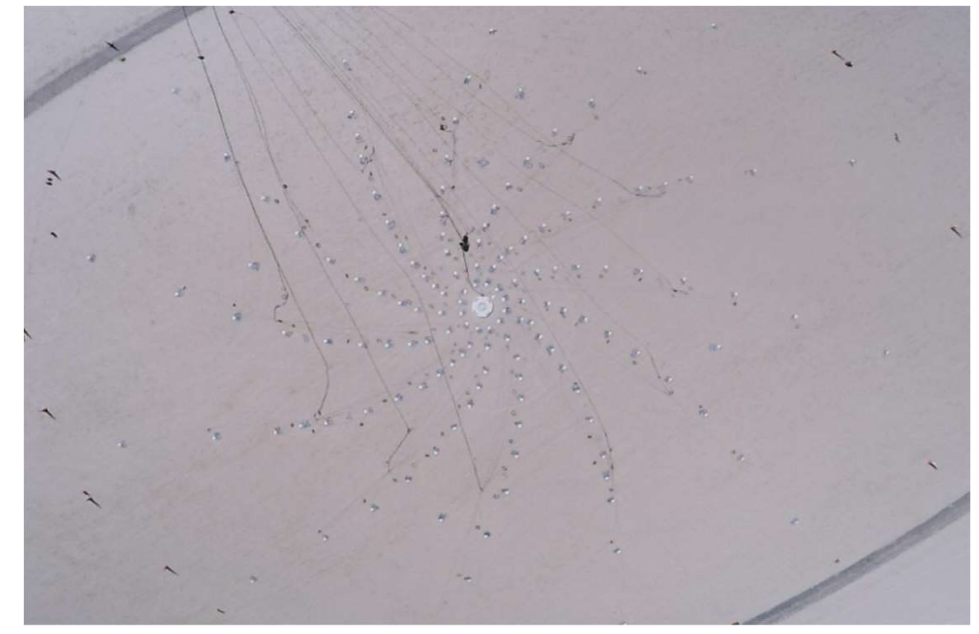

Figure 4. Aerial view of array deployment on Runway 18L, Edwards AFB.

\section{Calibration Methods}

Pre- and Post-Deployment Benchtop Calibrations: Absolute baseline sensitivities and frequency responses for the array microphones are measured via a bench top calibration of each sensor both before and after a deployment. The calibrations are conducted using a Bruel and Kjaer 4226 multifunction calibrator* as a precision sound source and a National Instruments USB 9215 digitizer to record both the reference signal from the calibrator and the output of the microphone (see Figure 5). Sensitivities are measured using a $250-\mathrm{Hz}, 94-\mathrm{dB}$ sound pressure level tone, corresponding to a pressure excitation of one Pascal at the microphone. Frequency response functions are measured by applying a broadband white noise signal to the external input port of the 4226 calibrator.

Figure 6 shows representative pre- and postdeployment histograms of the measured sensitivities for the ensemble of microphones fabricated for the array. The distribution of

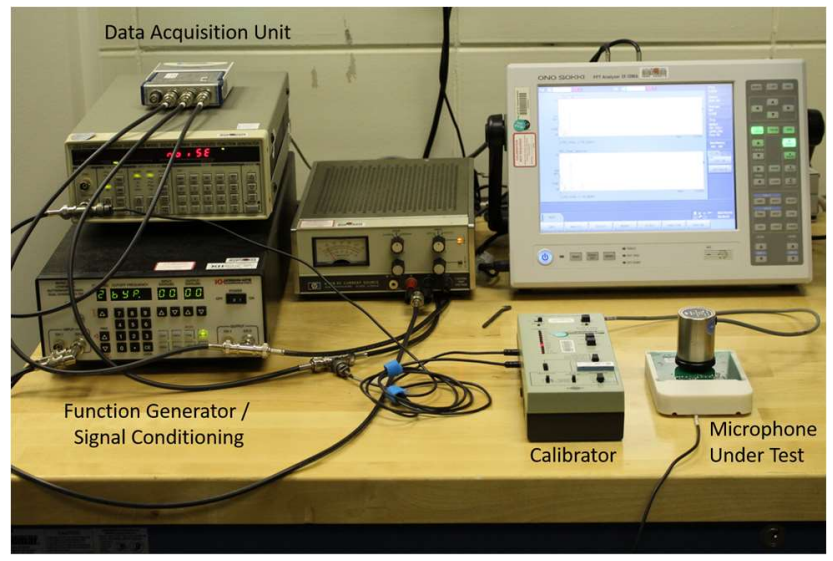

Figure 5. Benchtop calibration hardware.

\footnotetext{
* Specific vendor and manufacturer names are explicitly mentioned only to accurately describe the test hardware. The use of vendor and manufacturer names does not imply an endorsement by the U.S. Government nor does it imply that the specified equipment is the best available.
} 

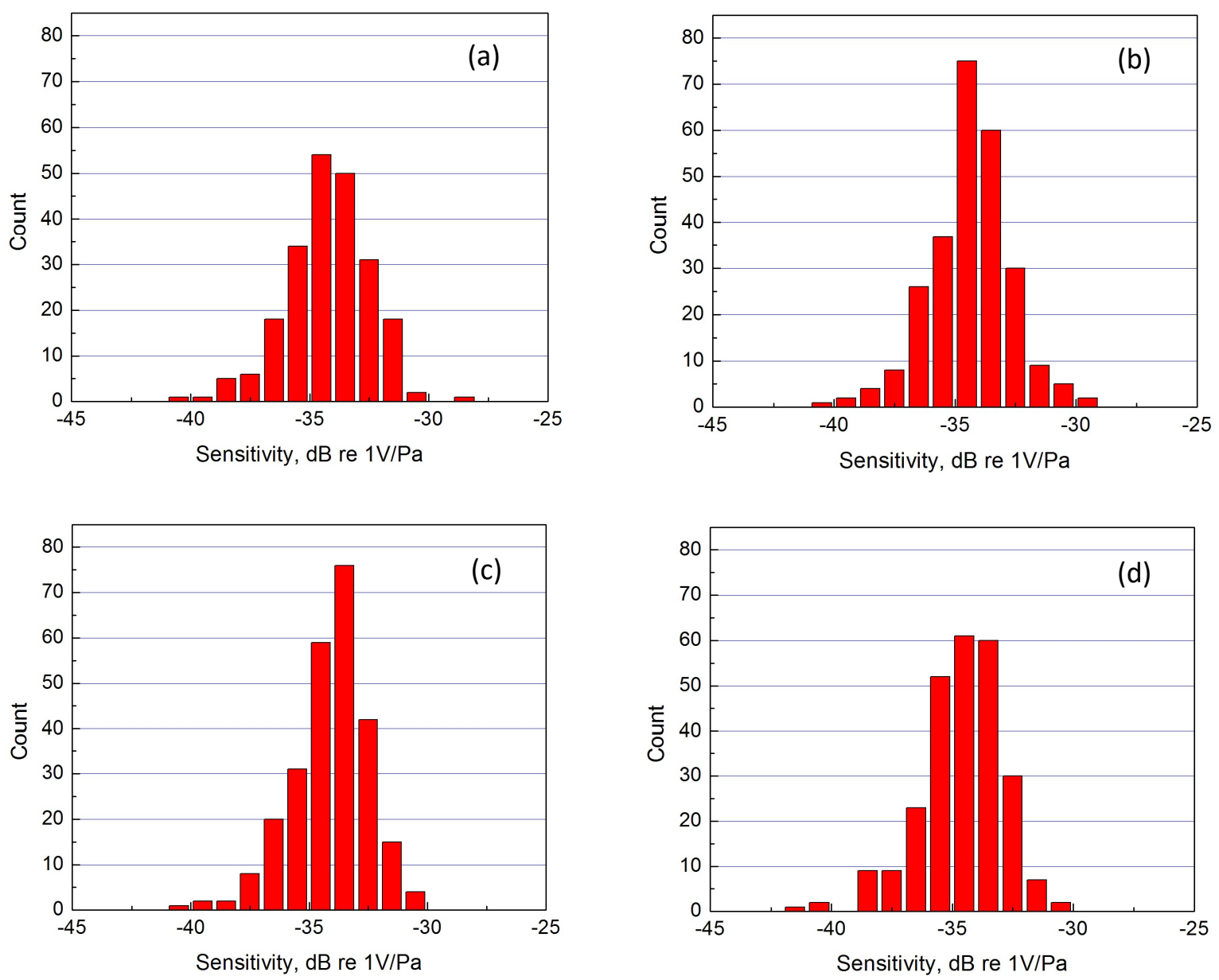

Figure 6. Histogram of measured microphone sensitivities.

(a) Pre A.P. Hill calibration, mean sensitivity $=-\mathbf{3 4 . 3 6} \mathrm{dB}$

(b) Post A.P. Hill calibration, mean sensitivity $=-\mathbf{3 4 . 5 5} \mathrm{dB}$

(c) Pre Edwards calibration, mean sensitivity $=\mathbf{- 3 4 . 2 6} \mathrm{dB}$

(d) Post Edwards calibration, mean sensitivity $=-34.72 \mathrm{~dB}$

sensitivities in each case is approximately Gaussian in shape with an overall mean sensitivity for all of the sensors of $18.8 \mathrm{mV} / \mathrm{Pa}(-34.5 \mathrm{~dB}$ re $1 \mathrm{~V} / \mathrm{Pa})$. Note that additional microphones were manufactured after the deployment at A.P. Hill, resulting in a change in shape in the computed histogram shown in Fig. 6b. The change in shape in the histogram shown in Fig. $6 \mathrm{~d}$ is speculated to be due to observed drifts in the sensitivities of individual microphones after the extended deployment at Edwards (a maximum drift of approximately $-1 \mathrm{~dB}$ was observed for a few sensors), with a corresponding change in the mean sensitivity for the ensemble. Note that these histograms are used in the selection criteria for choosing those microphones that will be used in the array. Sensors with sensitivities at the outer edges of the distribution are identified as spares and are not incorporated into the array unless needed.

Aerial Sound Sources: A unique in-situ calibration method for the array sensors was attempted for the first time during the Fort A.P. Hill and Edwards AFB deployments using a hovering aerial sound source. In principle, comparison of recorded microphone output levels during flights of the sound source allows the relative response drift of the sensors to be tracked on a daily basis, as long as the effects of speaker directivity, sound propagation (including meteorological conditions) and vehicle station keeping are taken 
into account. Two different sUAS vehicles were chosen for this purpose, denoted here as the Mark I and Mark II Acoustic Calibration Vehicles (ACV's). The salient features of each of these vehicles are summarized in Table I.

Table 1. Acoustic Calibration Vehicle Specifications.

\begin{tabular}{|c|c|c|}
\hline & Mark I ACV & Mark II ACV \\
\hline sUAS Type & Multi-Rotor, 6 Brushless Motors & Multi-Rotor, 8 Brushless Motors \\
\hline Manufacturer & Prioria Robotics & DJI \\
\hline Diagonal Length & 31 inches & 41.1 inches \\
\hline Maximum Weight & $16.6 \mathrm{lbs}$ & $24.3 \mathrm{lbs}$ \\
\hline Landing Rails & Fixed & Retractable \\
\hline Speed & $0-23$ knots & $0-42.5 \mathrm{knots}$ \\
\hline Endurance & 25 minutes & 15 minutes \\
\hline Command and Control & Remote Control Ground Station & Remote Control Ground Station \\
\hline Sound Source Payload & Anchor MiniVox Lite PA Speaker & Anchor MiniVox Lite PA Speaker \\
\hline
\end{tabular}

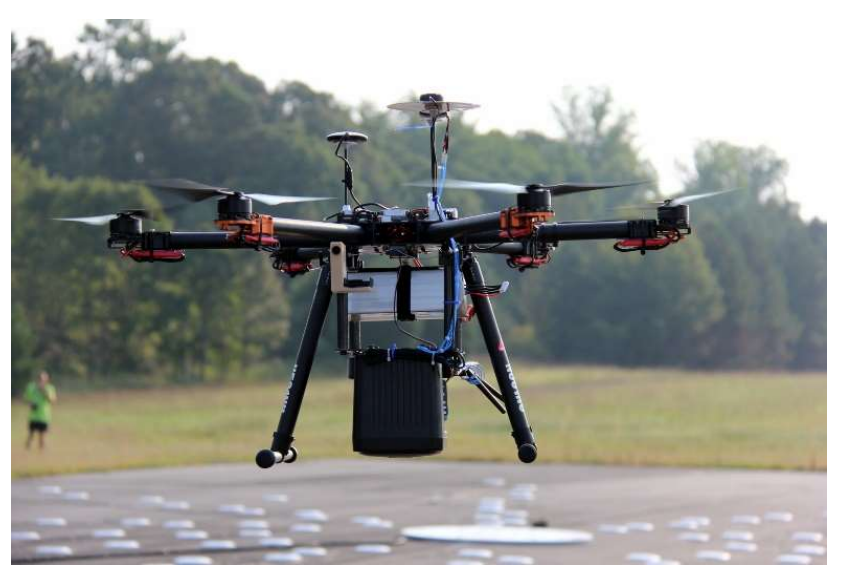

Figure 7. Mark I ACV at Fort A.P. Hill.

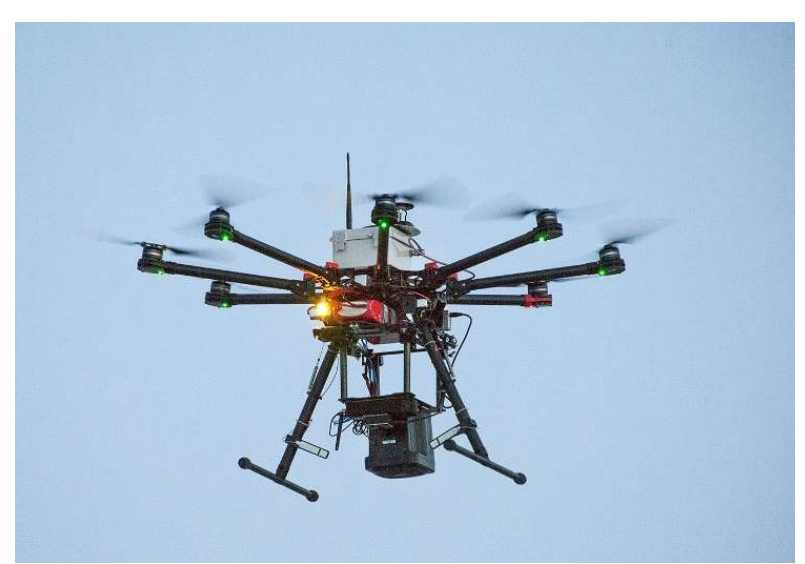

Figure 8. Mark II ACV at Edwards AFB.

The Mark I ACV consists of a Prioria Robotics Hex Flyer with a suspended battery-powered Anchor ${ }^{\circledR}$ MiniVox Lite public address speaker and MP3 player as the payload. The vehicle is shown in flight in Figure 7. The speaker provides both tonal and white noise excitation of the array at a number of vehicle altitudes spanning 50 to 400 feet. The Mark I ACV was utilized at Fort A.P. Hill with multiple daily flights conducted to provide a running measure of sensor responses.

The Mark II ACV consists of a DJI S1000 Octo Flyer with the same MiniVox speaker and MP3 player as the Mark I vehicle. The Mark II ACV, shown in flight in Figure 8, incorporates retractable landing rails (to eliminate acoustic scattering from the rails) and the ability to lift heavier payloads. A significant enhancement to the Mark II vehicle is the addition of a Real Time Kinematic (RTK), Global Positioning System (GPS) allowing three-axis vehicle location data to be obtained relative to the phased array center and accurate to 0.4 inches. Note that a modification to the MiniVox speaker housing was also implemented on the Mark II vehicle. This modification involved the replacement of the original bezel cover over the paper-cone speaker with a 3D-printed open cover. As will be shown, the modification eliminated sound scattering by the bezel. 
Ground Sources: In addition to the aerial sound source, a new complementary calibration method for response tracking was attempted during the Edwards AFB deployment. The method consists of embedding ground calibration speakers within the array and using these as fixed reference sound sources at precisely known locations. Several types of speakers were evaluated for their suitability for use as sound sources including horn drivers and domes. After testing several candidates, the Tymphany BC25SC06 domed tweeter was chosen as the source since it exhibits the best output levels with minimal distortion and with an omnidirectional response. Note that a further benefit of using a domed tweeter is that the speaker can be easily flush-mounted into the same type of ground plates used to house the array microphones. An example of one of the speakers mounted in a ground plate can be seen in Figure 9.

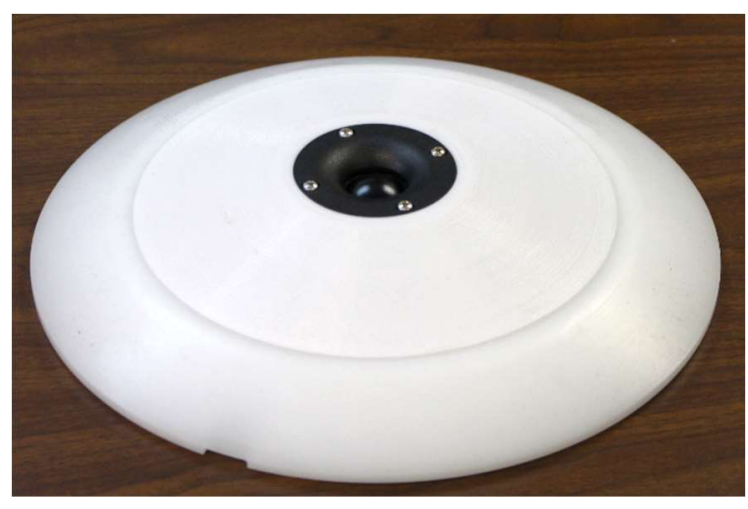

Figure 9. Tweeter mounted on ground plate.

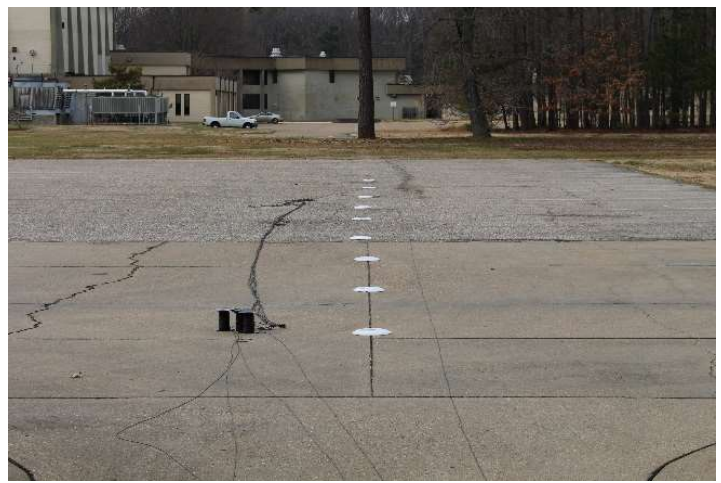

Figure 10. Risk-reduction testing of ground speaker to determine extent of influence on microphones.

Based on risk-reduction outdoor testing conducted at LaRC prior to the Edwards deployment (Figure 10), it was determined that acceptable time histories could be acquired by the array microphones under quiescent conditions up to 80 feet away from one of the ground sources when excited by a $2-\mathrm{kHz}$ tone. Accordingly, eight speakers were strategically placed in a circular pattern halfway between the center and outer edge of the Edwards array pattern. Speaker locations are depicted in Figure 11 (viewed from "behind" the array looking up).

To provide sensor response tracking, each speaker was energized in turn with a $2-\mathrm{kHz}$ tone with sufficient output power to excite the sensors without causing harmonic distortion in the speaker's output. Figure 12 shows typical ground footprints of local SPL levels for the speakers. The microphones excited by each speaker are denoted in the figure. Note that the 49 microphones in the central portion of the array (mounted on a common central mounting plate) are within 80 feet of all the speakers and thus are included in all calibration runs. In general, the footprints are very consistent for the various speakers, with the footprints for speakers 1,3 , 5 , and 7 located on the $\mathrm{x}$ and $\mathrm{y}$ axes of the array being slightly more compact due to their closer proximity to the array microphones (resulting in higher recorded SPL levels on the order of $5 \mathrm{~dB}$ ). 

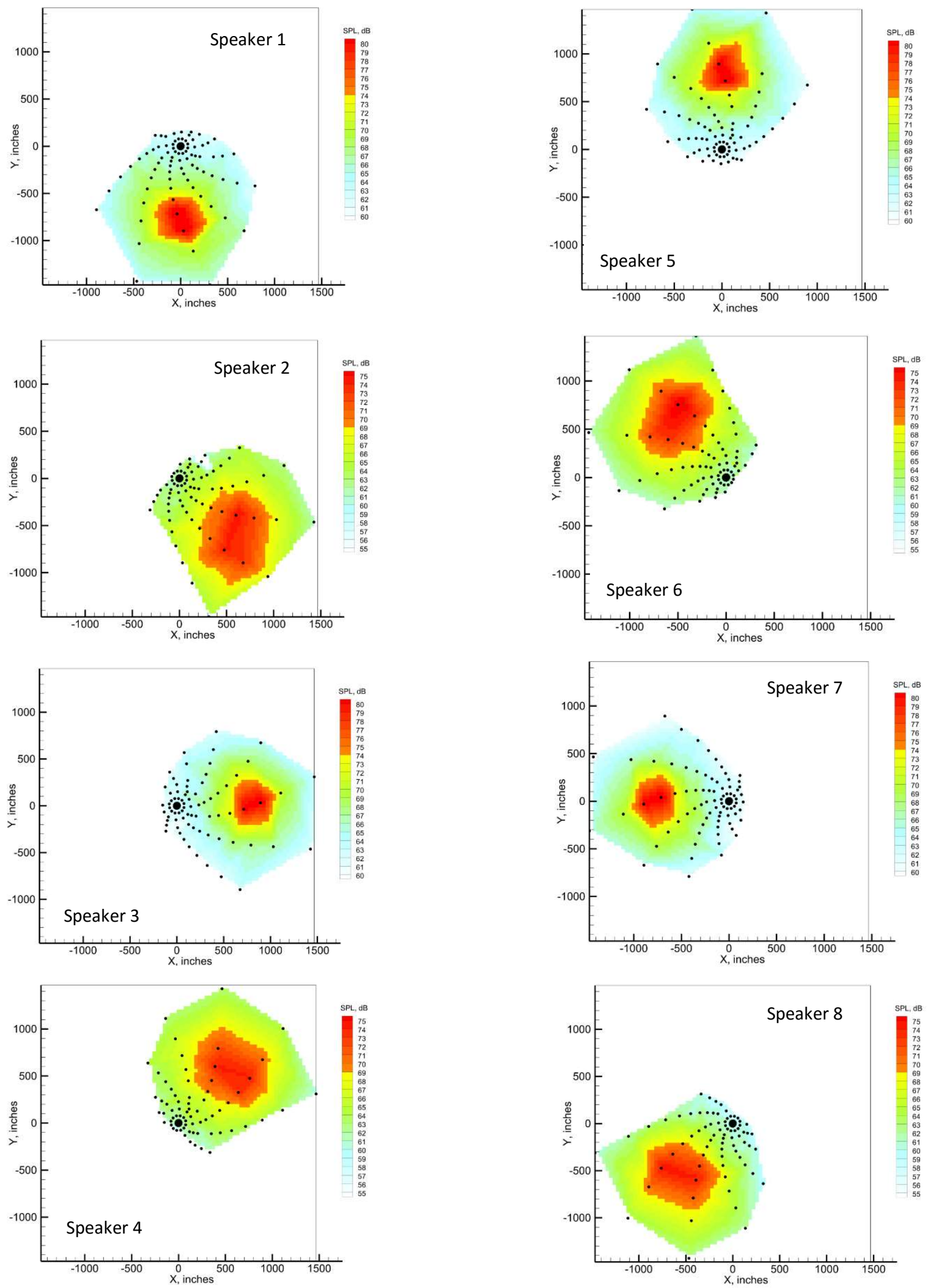

Figure 12. Nominal signal footprints for ground calibration speakers. The array microphones tracked by each speaker are shown.

7 of 22

American Institute of Aeronautics and Astronautics 


\section{Data Corrections and Strategy for Tracking of Microphone Responses}

Proper tracking of microphone responses using either aerial or ground source methods requires a number of corrections to be performed to the recorded microphone outputs. These corrections include accounting for the effects of the speaker directivity as a function of propagation angle and frequency, atmospheric attenuation and the $\mathrm{dB}$ loss of sound from spherical spreading between the source and each microphone. These corrections are described subsequently.

Corrections for Speaker Directivity: As described in Ref. 4, detailed characterizations of the aerial speakers utilized during the A.P. Hill and Edwards AFB deployments were performed in the LaRC Structural Acoustics Loads and Transmission (SALT) facility. Polar and azimuthal directivities as a function of frequency were measured for both the original speaker utilized on the Mark I ACV and the modified speaker utilized on the Mark II vehicle. Figure 13 shows the directivity functions for the two speaker configurations. As expected, removal of the bezel from the front of the Mark II ACV speaker results in a significant improvement in the structure of the directivity pattern at higher frequencies as seen in Figs. 13c and 13d.
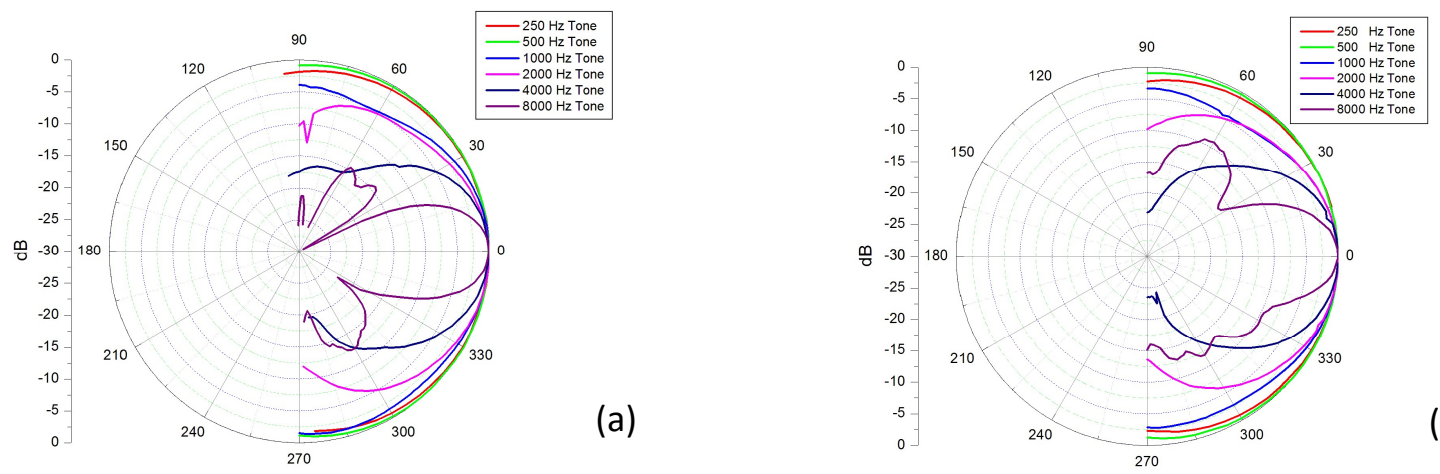

(b)
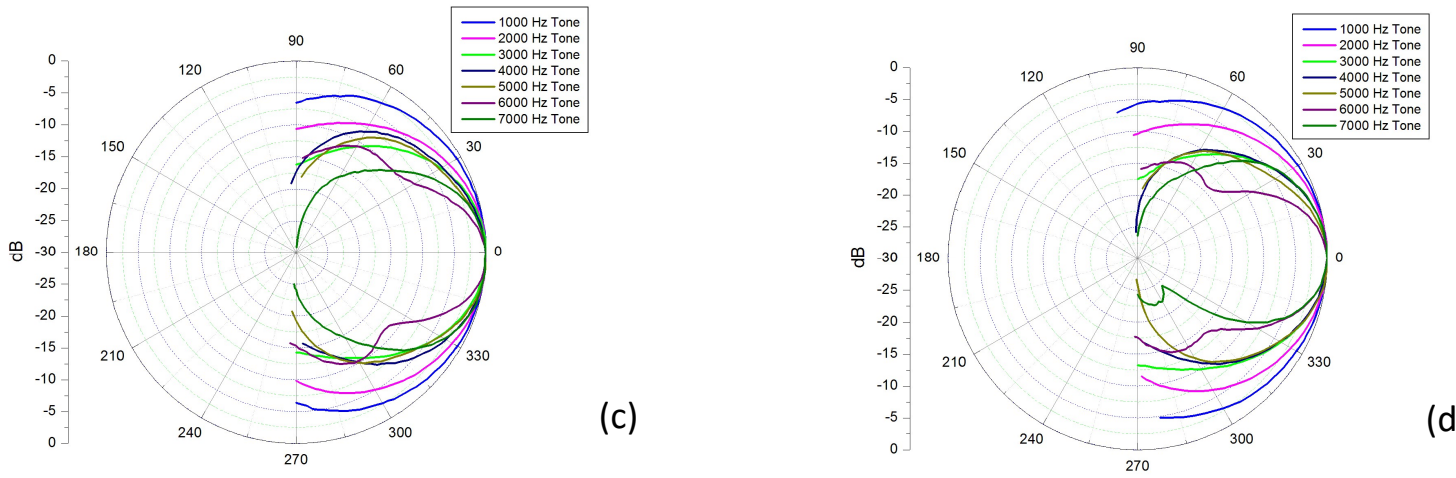

(c)

Figure 13. Aerial sound source directivity characteristics.

Polar (a) and azimuthal (b) directivity functions for Mark I ACV speaker. Polar (c) and azimuthal (d) directivity functions for Mark II ACV speaker. 
To predict the effects of the speaker directivity on measured sound pressure levels given the small array aperture deployed at A.P. Hill and the much larger one deployed at Edwards, the maximum dB loss at those angles corresponding to the edges of the aperture in the polar and azimuthal directions have been extracted from the functions in Fig. 13 and tabulated in Table II. Similar to the analysis shown in Ref. 4, the diameter of the array deployed at A.P. Hill was defined as 79 feet while the diameter of the Edwards array was defined as 250 feet. The vehicle altitudes were 400 and 350 feet for the ACV I and II vehicles, respectively.

Table II. Predicted dB Losses at Edge of Array Due to Sound Source Directivity

\begin{tabular}{|c|c|c|c|c|}
\hline & \multicolumn{2}{|c|}{ Mark I ACV Original Speaker } & \multicolumn{2}{c|}{ Mark II ACV Modified Speaker } \\
\hline & \multicolumn{2}{|c|}{$\begin{array}{c}\text { Aperture = 79 feet, Altitude = 400 feet } \\
\text { Aperture = 250 feet, Altitude = 350 feet }\end{array}$} \\
\hline $\begin{array}{c}\text { Frequency } \\
(\mathbf{H z})\end{array}$ & Polar dB Loss & $\begin{array}{c}\text { Azimuthal dB } \\
\text { Loss }\end{array}$ & Polar dB Loss & $\begin{array}{c}\text { Azimuthal dB } \\
\text { Loss }\end{array}$ \\
\hline 1000 & 0.1 & 0.2 & 0.4 & 0.4 \\
\hline 2000 & 0.2 & 0.2 & 1.0 & 0.8 \\
\hline 4000 & 0.3 & 0.1 & 1.9 & 1.6 \\
\hline 7000 & N/A & N/A & 2.9 & 1.5 \\
\hline 8000 & 0.6 & 0.6 & N/A & N/A \\
\hline
\end{tabular}

An examination of the loss figures in Table II reveals that operating the speaker with a 4-kHz tone represents a good compromise between maintaining a smooth directivity function and providing enough speaker output (based on the manufacturer's published frequency response), while helping to properly separate the speaker tone from the ACV vehicle noise. Thus, all of the tracking data shown in Section IV for the Mark I and II aerial sources were obtained using a 4-kHz tone. As seen in Fig. 13, there is very little difference between the directivity functions in the polar and azimuthal directions at this frequency. Thus, a simplified approach was taken for computing a correction where it was assumed that the overall directivity function was hemispherical in structure. Thus, for individual microphones, the mean of the polar and azimuthal functions at a single directivity angle was used in computing an average $\Delta d B$ quantity to add to the microphone output:

$$
\begin{gathered}
\Delta d B_{i}=\frac{P_{\theta}\left(\alpha_{i}\right)+P_{\varphi}\left(\alpha_{i}\right)}{2} \\
\alpha_{i}=\sin ^{-1}\left(\frac{r}{d}\right)
\end{gathered}
$$

where $P_{\theta}$ is the polar directivity function as shown in Fig. 13, $P_{\varphi}$ is the azimuthal directivity function, $\alpha_{i}$ is the directivity angle for the $\mathrm{i}^{\text {th }}$ microphone in the array, $r$ is the distance from the microphone to the spot on the ground directly under the ACV (as reported by the on-board GPS tracking), and $d$ is the line-of-sight distance from the aerial source to the microphone.

Although it was not measured in the LaRC SALT facility, it is assumed that the 90-degree polar and azimuthal directivity functions for the Tymphany tweeter ground speakers at Edwards are uniform based on manufacturer's response data for these devices. Given that the speakers are placed flat on the ground and incorporate a hemispherical dome structure, it is safe to assume that the directivity should be omnidirectional to all of the microphones in the array within an 80 -foot radius to the speaker. Thus, no corrections for directivity were performed for these speakers.

Corrections for Atmospheric Attenuation: Two different corrections for atmospheric attenuation were performed depending on whether an aerial or ground source was utilized for microphone response tracking as well as whether atmospheric temperature and humidity profiles were available. For aerial source measurements where weather profiles were available, a layered correction approach was employed similar to that described by True et al. ${ }^{5}$ This correction stratifies the atmosphere into $h=20$-foot layers extending 
from the ground to the sound source on the ACV (see Figure 14). The local atmospheric attenuation is computed for each layer based on the average temperature and humidity within the layer as measured by a weather profiling system (e.g., a radiosonde on a balloon). Within a layer, the distance along the line of sight connecting the location of the ACV (from the GPS tracking) and an array microphone was used to compute the total $\mathrm{dB}$ loss of sound within the layer. These separate $\mathrm{dB}$ losses were then summed to obtain the total loss from the sound source to the microphone. The total correction to add to an individual microphone's output as a function of source frequency is given by

$$
d B_{\text {total }, \text { mic }}(f)=\sum_{i=1}^{n} d B_{i, m i c}\left(f, T_{i}, H_{i}, d_{i}\right)
$$

where $f$ is the source frequency and $d B_{i, m i c}$ is the computed $\mathrm{dB}$ loss across the $\mathrm{i}^{\text {th }}$ atmospheric layer (with average temperature $T_{i}$ and humidity $H_{i}$ over a distance $\left.d_{i}=h / \cos (\xi)\right)$ within a total of $n$ layers. The atmospheric attenuation for each layer was determined according to ANSI standard S1.26-1978. ${ }^{6}$

For ground and aerial source runs where a weather profile was not available, it was assumed that the atmosphere was uniform in temperature and humidity from the sound source to a microphone. Thus, only a bulk atmospheric attenuation and associated $d B_{\text {total,mic }}$ loss correction were computed and applied to the array microphones.

Response Tracking Strategy: The overall strategy for tracking the response of individual microphones in the array is to establish an initial baseline output for each sensor. Deviations from this baseline are then identified via subsequent calibration runs using a combination of aerial and ground sound sources. If a microphone needs to be replaced in the array, then a new baseline is established at that point in time for the replaced sensor. The following procedures were developed to acquire time history data from the array in order to subsequently identify microphone response deviations from baseline levels and/or sensor or channel failures (occurring at any point in the sensor / data acquisition chain):

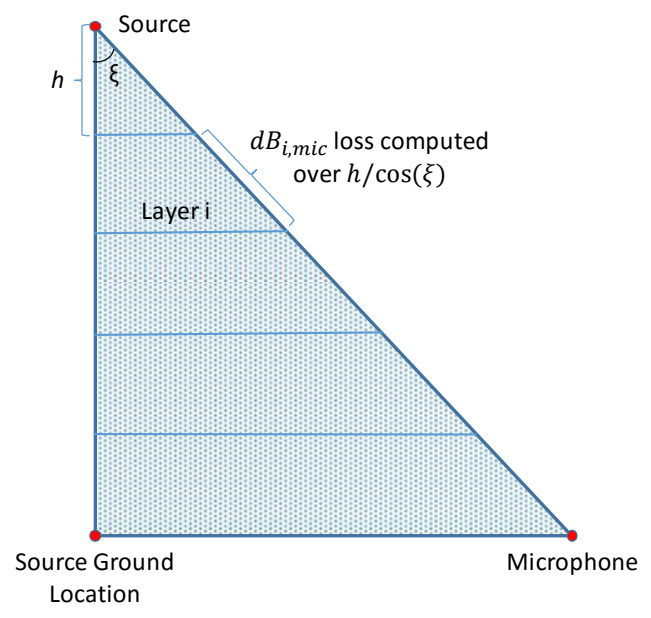

Figure 14. Layered atmospheric attenuation correction geometry.

\section{Aerial Sources}

1. The sound source on the ACV was programmed to produce a $4-\mathrm{kHz}$ tone and the vehicle was launched, centered over the array, and brought to the designated altitude (either 400 feet at A.P. Hill or 350 feet at Edwards AFB).

2. Time history data was captured for all of the array microphones over a nominal 10 -second recording cycle.

3. For the initial run, the pressure squared values for each microphone were computed at a $4-\mathrm{kHz}$ narrowband frequency and the equivalent sound pressure level (SPL) was calculated. Full corrections for speaker directivity and atmospheric attenuation as described previously were applied to the SPL values. This defined the baseline SPL for each microphone.

4. Absolute output changes across the array due to speaker drift as a function of time must be taken into account. Thus, for subsequent aerial source runs, the current microphone outputs had a reference level, $d B_{\text {ref }}$, subtracted from them. $d B_{\text {ref }}$ was obtained via one of two methods depending on the location of a microphone in the array: (1) for those sensors located within 24 feet of the center of the array, a mean $d B$ was computed for the inner 49 microphones in the array with $d B_{r e f}$ defined as the difference 
between the means for the current and baseline acquisitions, or (2) for those sensors located outside the 24-foot radius from the center, the mean was computed from the nearest 8 neighboring microphones, again with $d B_{\text {ref }}$ defined as the difference between the means for the current and baseline acquisitions.

5. Each subsequent calibration produced a $\Delta d B_{\text {mic }}$ by subtracting the baseline level from the current calibration via

$$
\Delta d B_{\text {mic }}=\left(d B_{\text {mic }}-d B_{\text {ref }}\right)_{\text {calibration }}-\left(d B_{\text {mic }}-d B_{\text {ref }}\right)_{\text {baseline }}
$$

A running history of the $\Delta \mathrm{dB}$ levels was maintained to observe trends and track the microphone responses.

\section{$\underline{\text { Ground Sources }}$}

1. Each of the 8 speakers embedded in the array pattern were excited individually using a 2-kHz tone.

2. For each speaker excitation, time history data was captured for all of the array microphones over a nominal 10-second recording cycle.

3. Baseline SPL levels and a running history of subsequent $\Delta d B_{\text {mic }}$ levels were obtained in the same manner as described previously in steps (3) - (5) for the aerial sources.

Two additional considerations needed to be addressed as part of the response tracking strategy. First, for the ground sources at Edwards, extreme changes in temperature encountered on the runway during the course of the deployment needed to be taken into account. Aeroacoustic flyover testing at Edwards normally commences around sunrise when the atmosphere is relatively calm and continues until surface heating either: (1) reduces the humidity below a minimum threshold, or (2) increases the winds above a maximum threshold. During this period, the temperature at the surface can vary as much as 30 degrees F. This modifies the output of the ground speakers due to an effect known as speaker thermal compression. ${ }^{7}$ As seen in Figure 15 with data obtained at Edwards on two different days, a significant drop in speaker SPL was observed as the temperature increased. It is difficult to correct for this effect; therefore, tracking of microphone responses using the ground speakers was only performed for runs obtained at matched temperatures (i.e., normally the first run of the

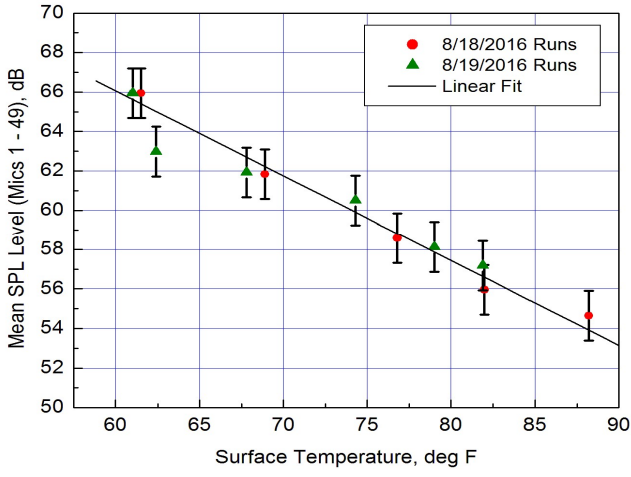

Figure 15. Effect of thermal speaker compression on observed output level. morning where temperatures were somewhat uniform day to day). Note that the aerial speakers utilized for calibration runs did not exhibit noticeable thermal effects due to better ventilation under the ACV which limited the heating of the speaker. The speakers on the runway surface were encased in ground plates without ventilation and thus experienced much higher temperatures.

The second consideration to be addressed concerned the effect of surface winds on the inter-channel coherence observed between microphone time histories for both aerial source and ground source calibration runs. An examination of multiple coherence functions revealed that the best tracking performance occurred for those runs exhibiting the highest consistent coherence between microphones. The high inter-channel coherence also correlated very well with lower average surface wind speeds (regardless of wind direction). Therefore, tracking of microphone responses for both aerial and ground source runs only occurred for those runs where the average wind speed did not exceed a defined maximum limit of $5 \mathrm{feet} / \mathrm{sec}$. 


\section{Tracking Examples and Evaluation of Methods}

Fort A.P. Hill Aerial Source: The first example of microphone response tracking and array health monitoring occurred at Fort A.P. Hill for aerial sound source measurements obtained using the Mark I $\mathrm{ACV}$. These are shown in Figures 16 and 17 for measurements conducted over a 7-day period where a total of 8 individual runs satisfied the tracking strategy given in Section III. Sixteen representative microphones were selected for inclusion in these figures, with sensors taken from the following regions of the array aperture: (1) four microphones on the central mounting plate, (2) four microphones located near the central plate, (3) four microphones located midway along the starburst arms of the array, and (4) four microphones located at the outer limits of the aperture. These selected microphones spanned all four quadrants of the array. The $\Delta d B$ values for a particular sensor have been normalized by the baseline level taken from the first calibration run. The error bars represent $95 \%$ confidence intervals based on the identification of the spectral peaks in the auto-spectra used to generate the tracking data. All of the autospectra were generated using Welch's periodogram method. ${ }^{8}$

As seen in Figs. 16 and 17, the majority of the microphones in the array maintained fairly uniform output levels as a function of time during the deployment. However, a few sensors did exhibit problems. In particular, microphone \#93 (Fig. 17e) showed a marked 3dB drop in output after the first day of the deployment. This is characteristic of a partial sensor failure, and although this sensor was not replaced in the array during the A.P. Hill deployment, the drop in output shown by the tracking data would be sufficient justification for a sensor replacement. Similarly, microphone \#3 (Fig. 16b) and microphone \#125 (Fig. 17b) show drops in output toward the end of the deployment that might be indicative of partial sensor failures as well. A couple microphones displayed some variability in the tracking (microphone \#185 in particular in Fig. 17h); however, it is not as apparent from this data whether there are any problems occurring with these sensors. In general, the aerial sound source calibrations worked very well during the A.P. Hill deployment.

Edwards AFB Ground Sources: The second example of tracking the array response and health occurred at Edwards AFB for ground source measurements obtained over a period from August 18 - October 5, 2016. During this time, a total of 13 individual runs satisfied the tracking strategy given in Section III (i.e., matched temperatures and wind speeds below $5 \mathrm{feet} / \mathrm{sec}$ ). In a similar approach to that shown for the Fort A.P. Hill data, sixteen representative microphones were selected from the array aperture covering both inner, middle, and outer portions of the aperture across all four quadrants. These selected microphones are shown in Figures 18 and 19. The speaker used to excite the microphone is denoted on each figure. Note that gaps appearing in the tracking data in individual figure panels represent outliers that were identified and removed using a modified Thompson-tau method. ${ }^{9}$

In general, the ground source calibrations did a good job of providing response tracking information for the array sensors. Most of the microphones in Figs. 18 and 19 show only a minimal drift in response over the almost two-month period they were deployed at Edwards. A couple microphones (\#108 and \#106) did exhibit an approximate $+2 \mathrm{~dB}$ drift toward the end of the deployment. Although not shown in the figures, a few failed sensors were identified from their response tracking during the course of the deployment and replaced.

An interesting observation was that the day to day variability in the tracking was more pronounced for those sensors located near the edge of the array aperture versus those situated near the center of the array. Indeed, the majority of outliers removed from the data occurred for those microphones in the middle to outer portions of the array. The reason for the increased variability in tracking in the outer regions of the array aperture is not well understood yet. It is speculated that ground scattering effects may create variable sound propagations to individual microphones. Also, there may be deviations from the assumed uniform 90-degree directivity function as the sound propagates from the tweeters to the individual microphones. 


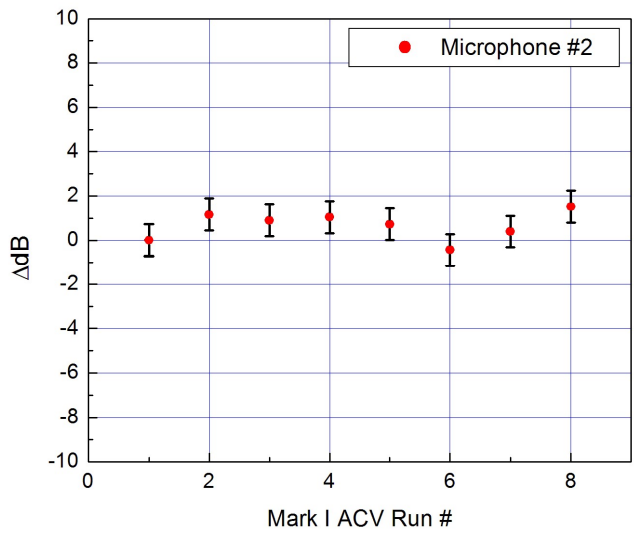

(a)

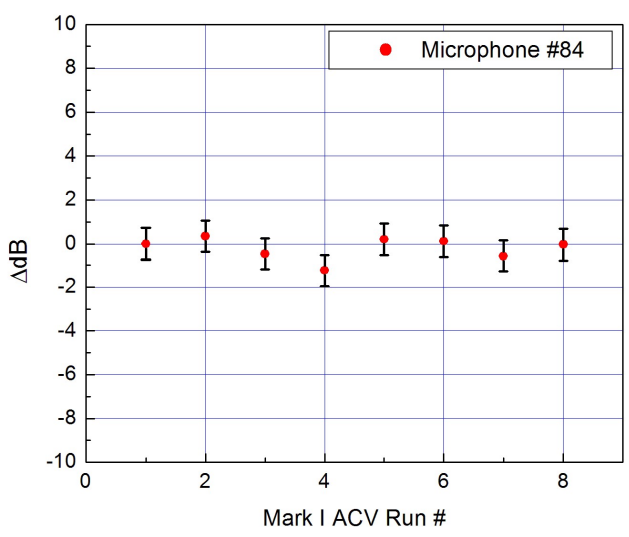

(e)

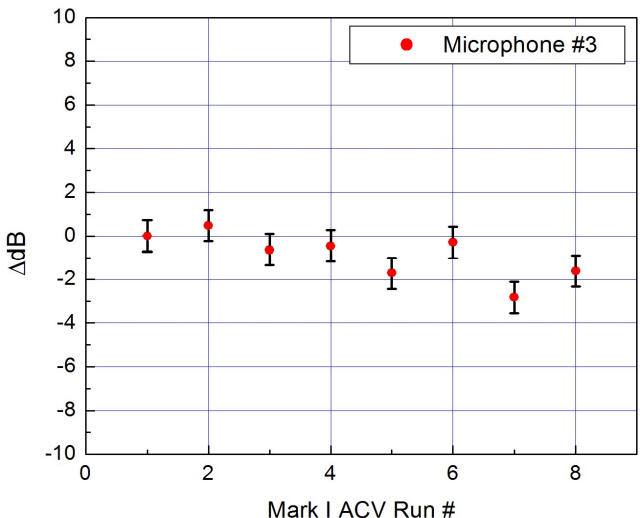

(b)

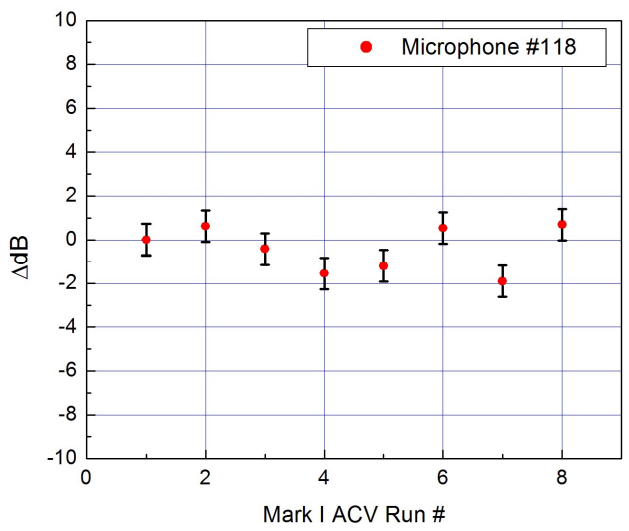

(f)

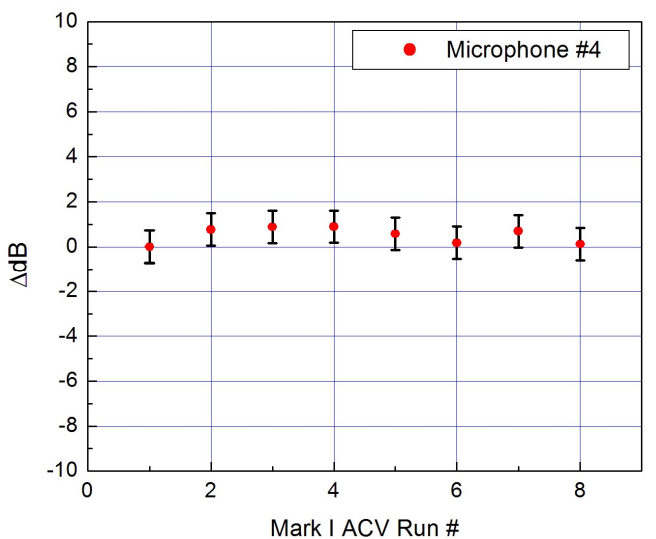

(c)

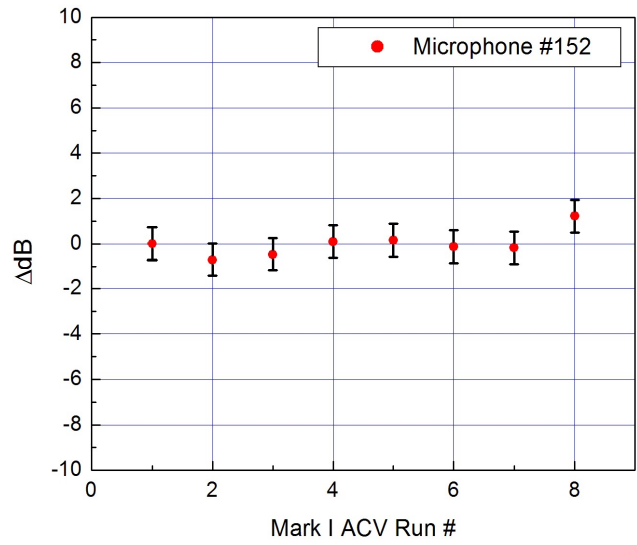

(g)
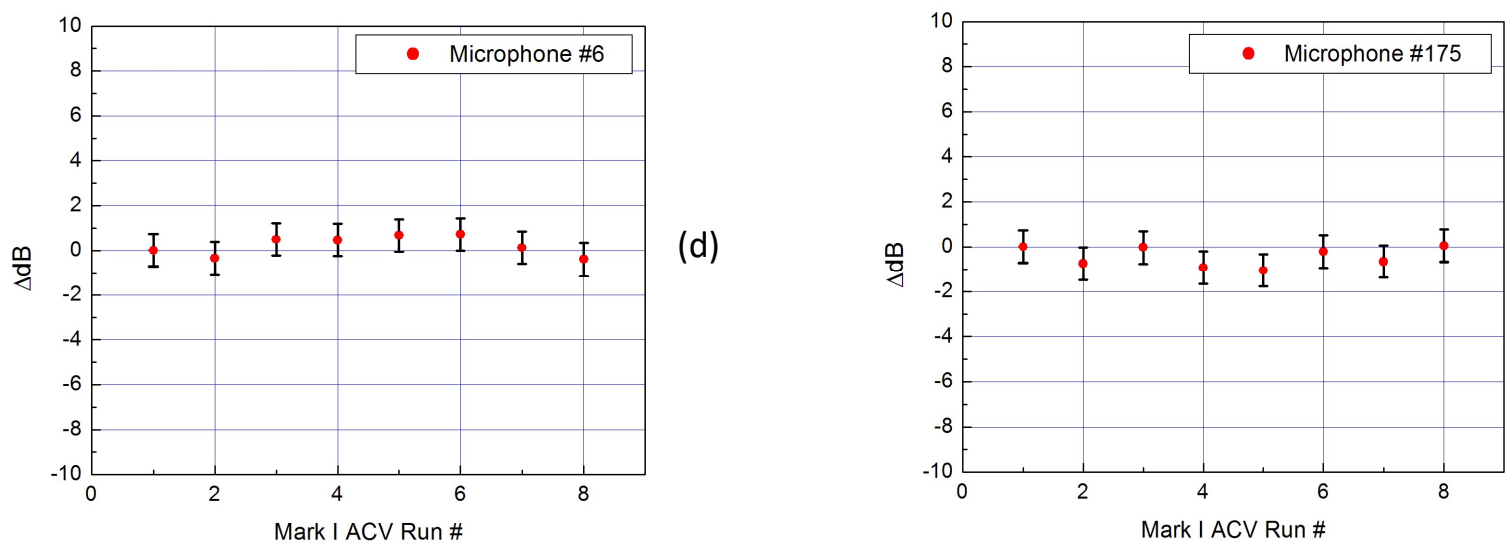

(h)

Figure 16. Tracking of measured microphones levels for 8 separate Mark I ACV runs conducted at Fort A.P. Hill over a 7-day period spanning August 27 - September 3, 2015.

(a) - (d) are microphones on central plate, (e) - (h) are individual inner microphones

13 of 22

American Institute of Aeronautics and Astronautics 


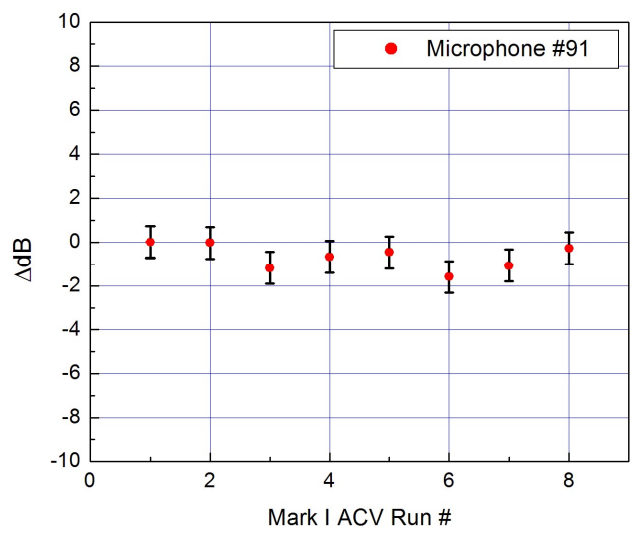

(a)

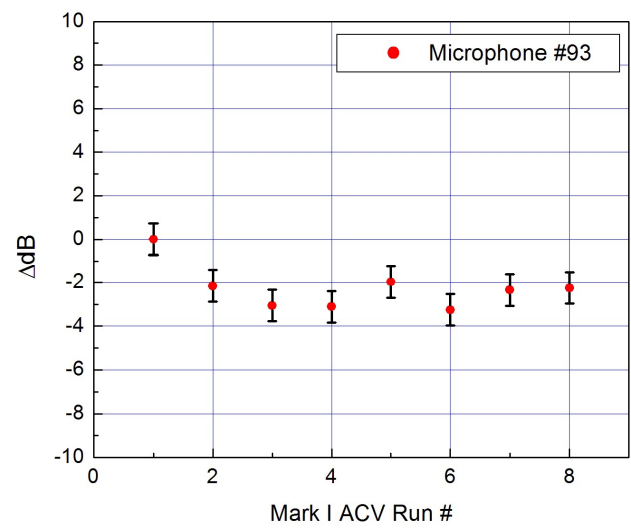

(e)

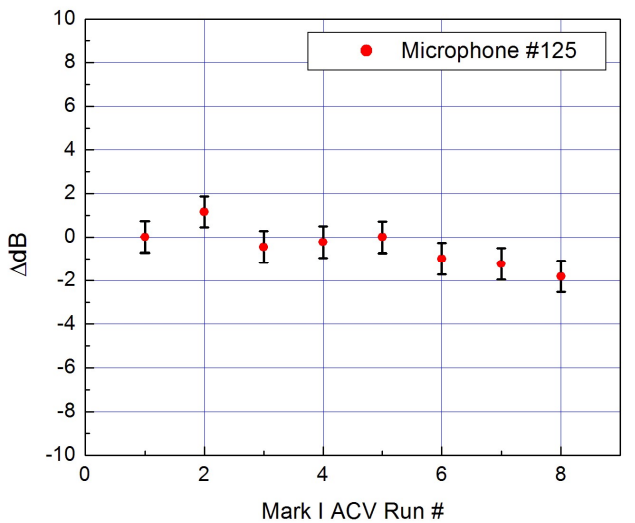

(b)

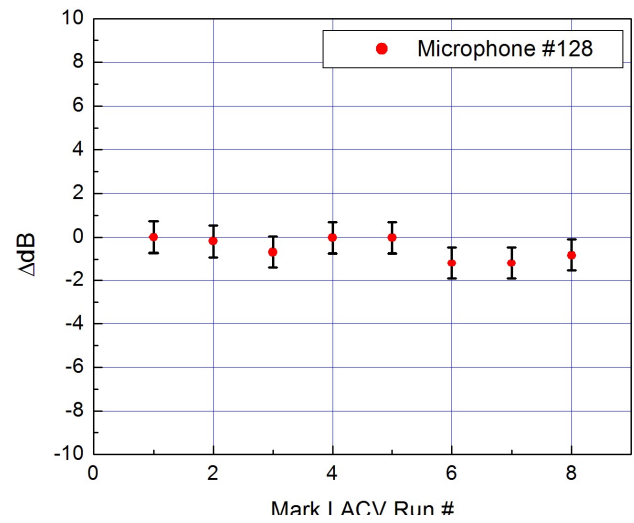

(f)

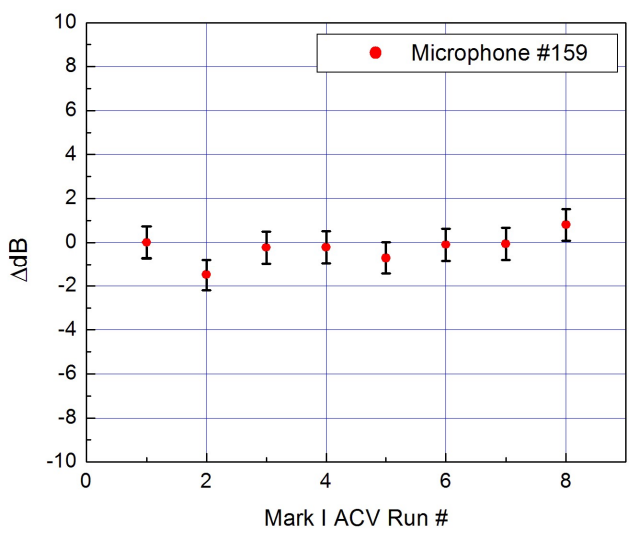

(c)

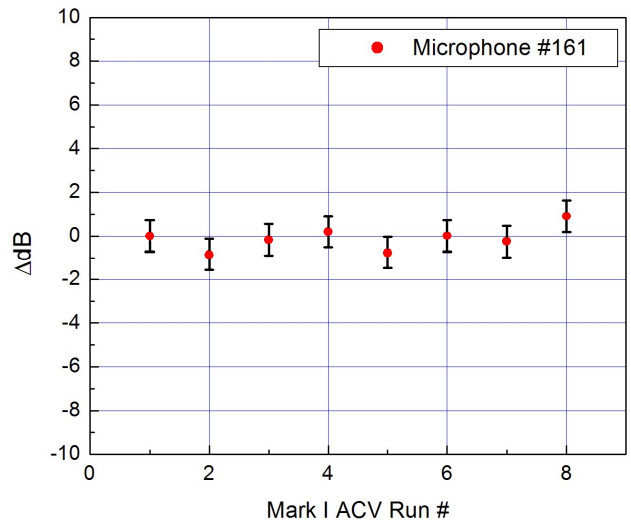

(g)
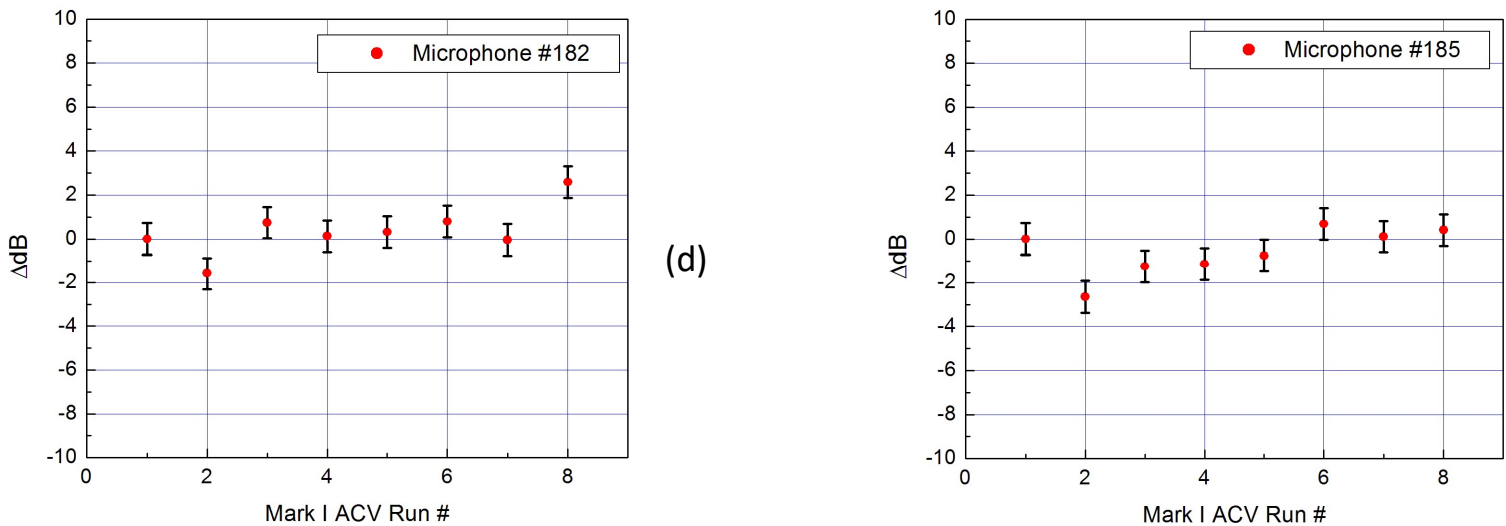

(h)

Figure 17. Tracking of measured microphones levels for 8 separate Mark I ACV runs conducted at Fort A.P. Hill over a 7-day period spanning August 27 - September 3, 2015.

(a) - (d) are individual middle microphones, (e) - (h) are individual outer microphones 


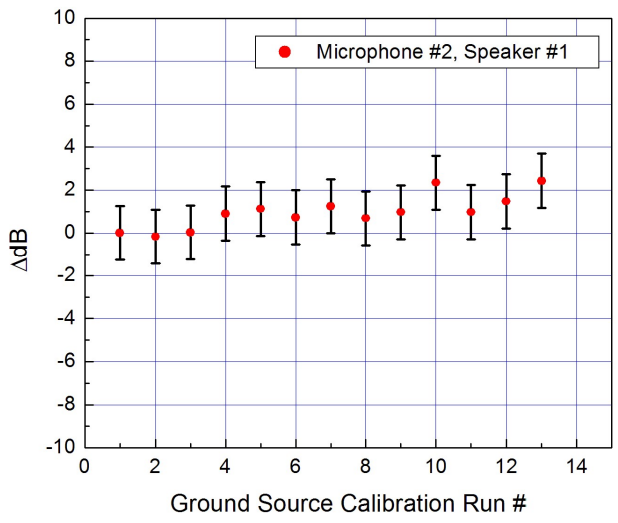

(a)

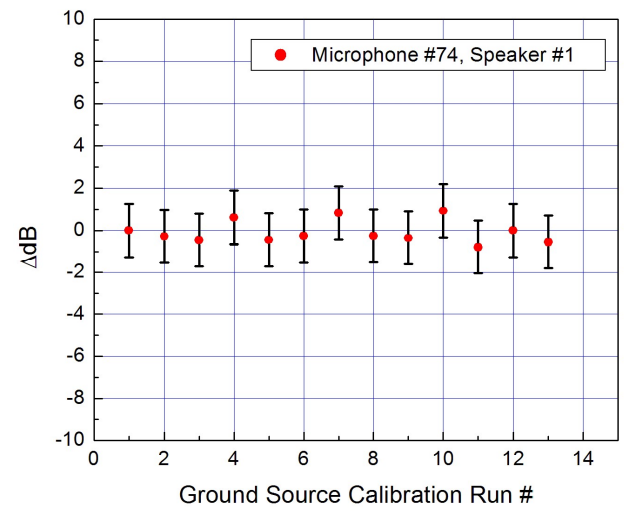

(e)

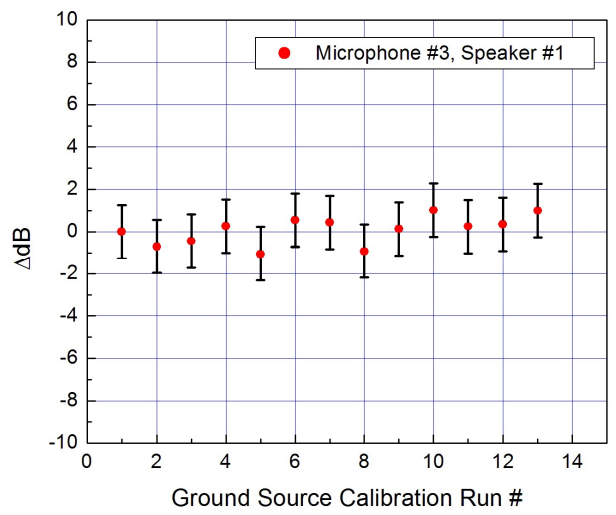

(b)

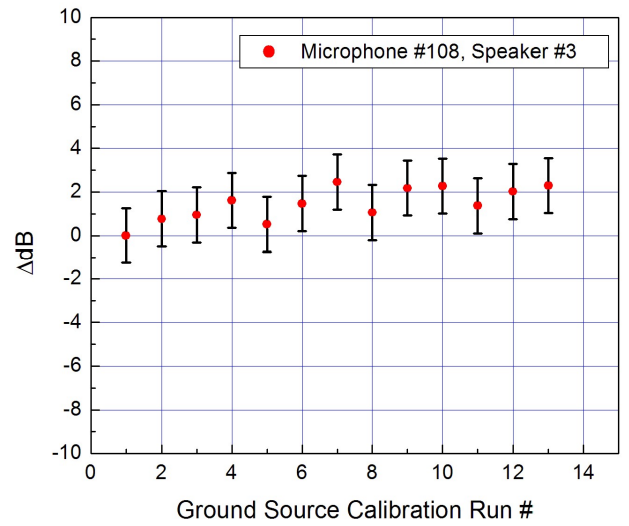

(f)
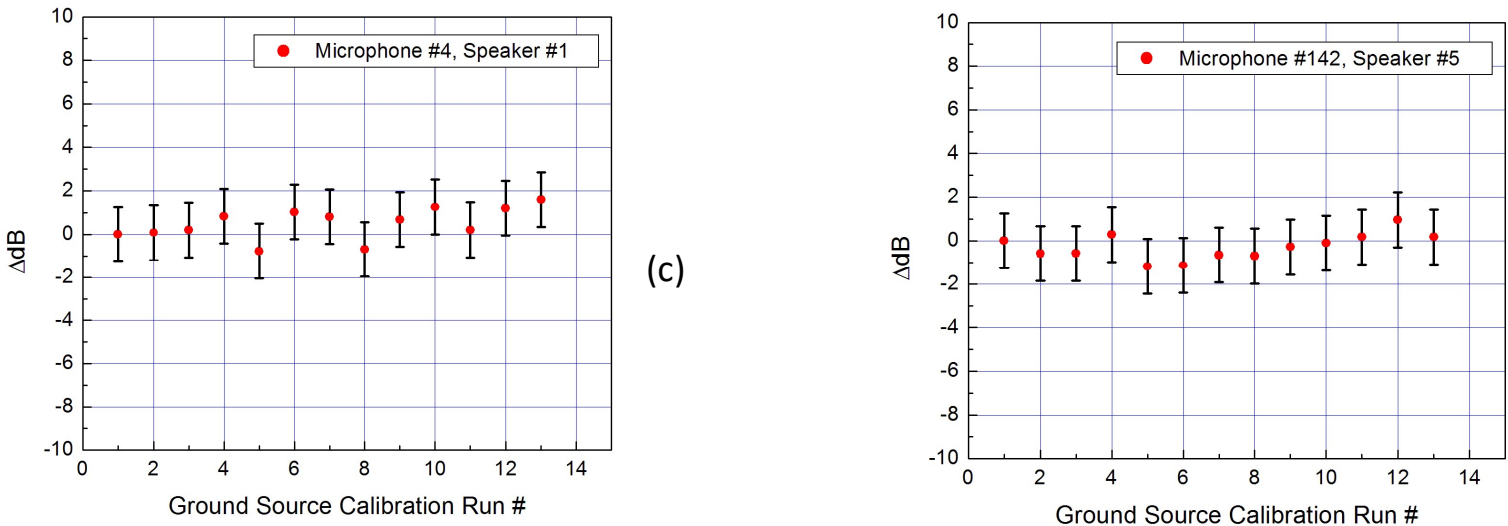

(g)
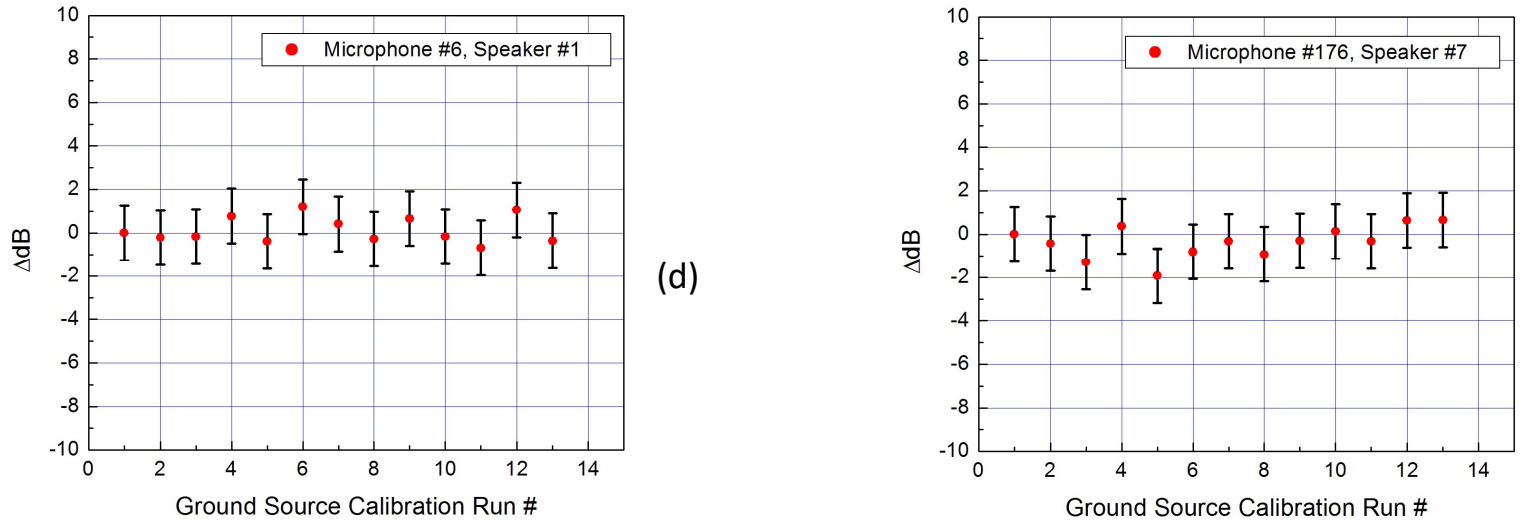

(h)

Figure 18. Tracking of measured microphone levels for 13 separate ground source runs conducted at Edwards AFB from August 18 - October 5, 2016.

(a) - (d) are microphones on central plate, (e) - (h) are individual inner microphones 15 of 22

American Institute of Aeronautics and Astronautics 


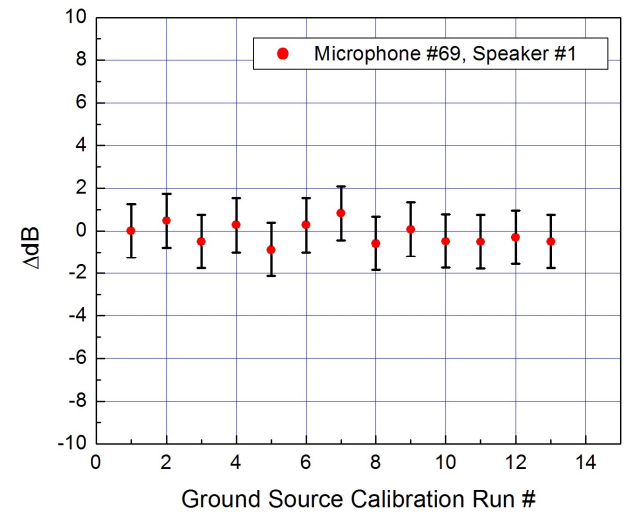

(a)

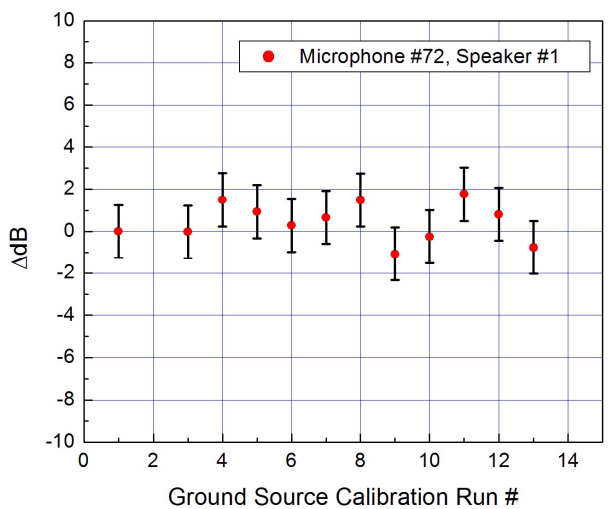

(e)

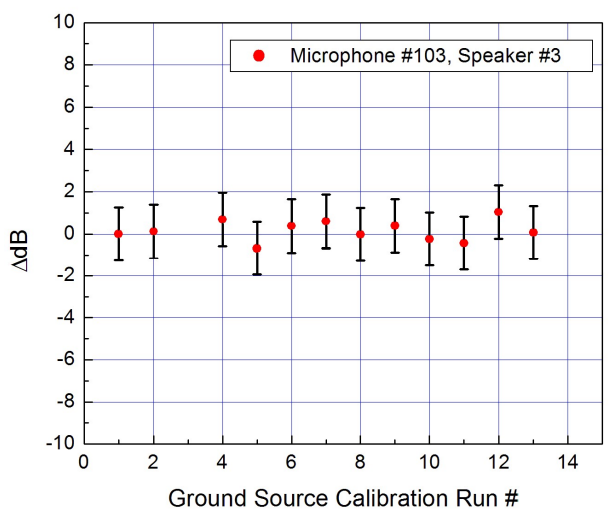

(b)

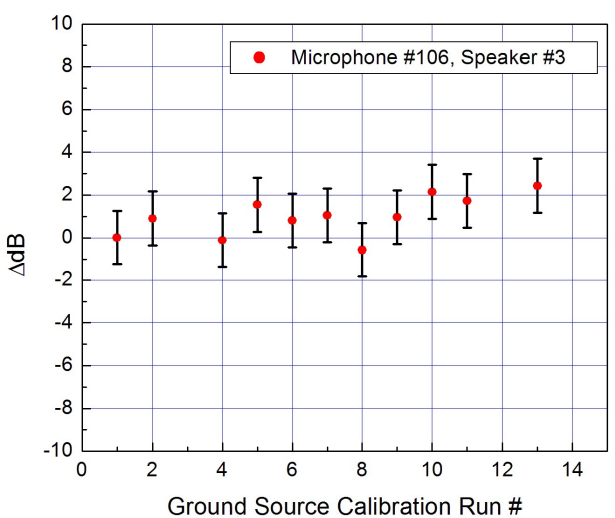

(f)
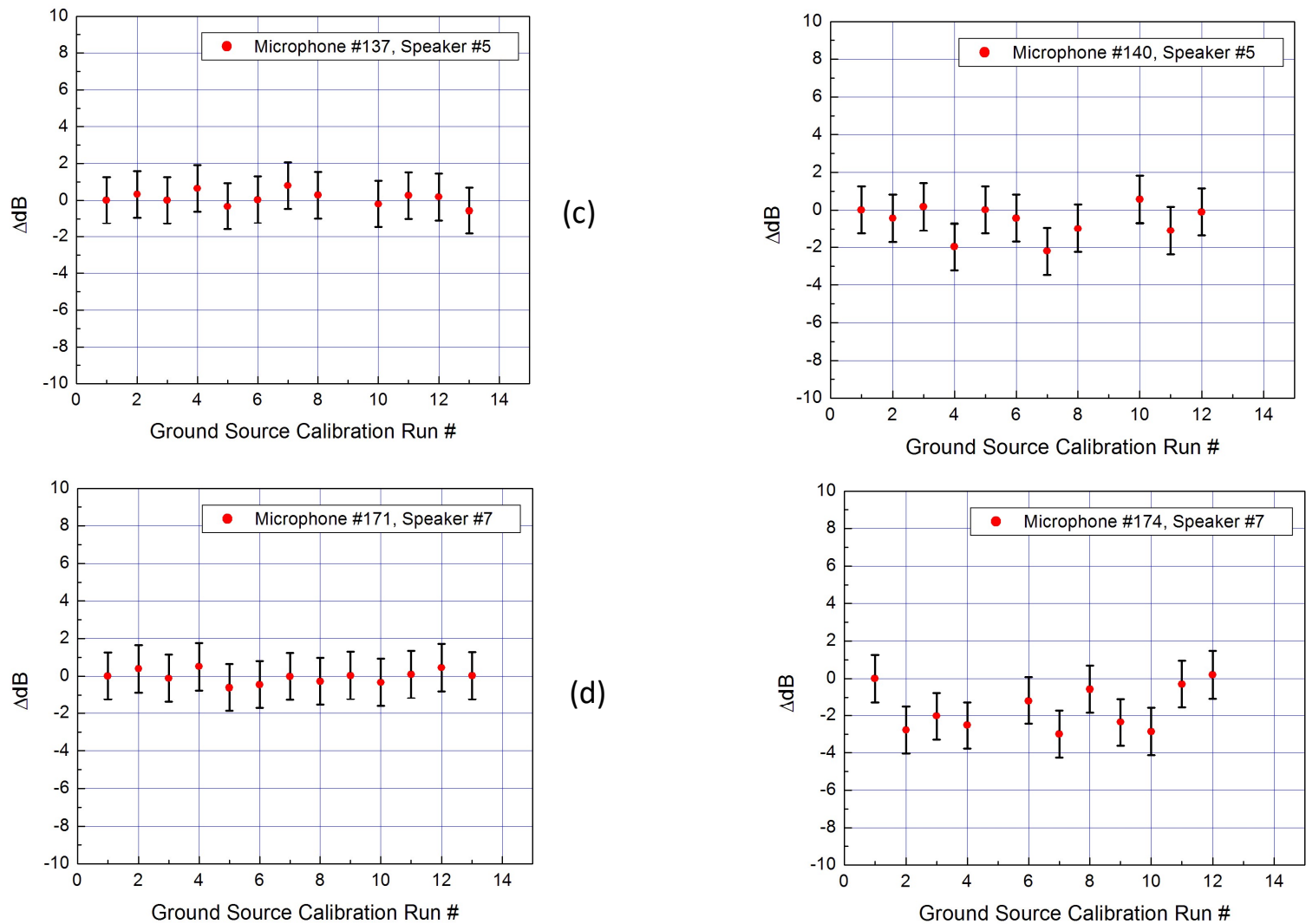

(c)

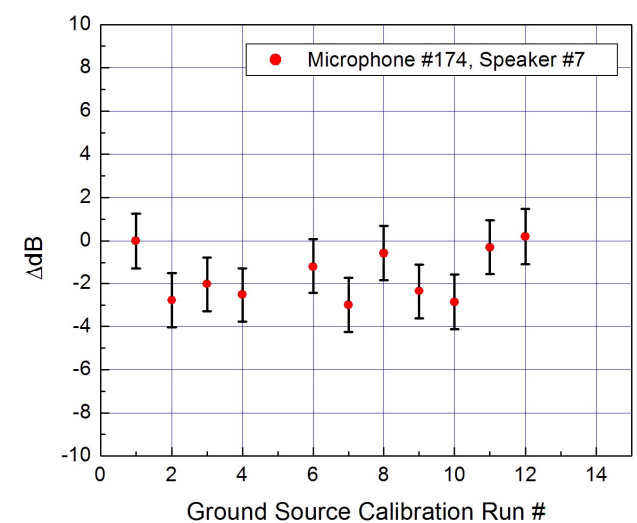

(g)

(h)

Figure 19. Tracking of measured microphone levels for 13 separate ground source runs conducted at Edwards AFB from August 18 - October 5, 2016.

(a) - (d) are individual middle microphones, (e) - (h) are individual outer microphones 
Edwards AFB Aerial Sources: A final example of tracking array microphone response also occurred at Edwards, using Mark II ACV aerial sound source calibrations conducted from August 18 - October 4, 2016. During this time, a total of 12 individual runs both satisfied the tracking strategy described in Section III (i.e., wind speeds below $5 \mathrm{feet} / \mathrm{sec}$ ) and matched same-day ground speaker runs so that comparisons between the two methods could be performed. The aerial sound source results are shown in Figures 20 and 21. The representative microphones selected from the array are the same as those shown in Figs. 18 and 19. Outliers in the tracking data were identified and removed.

In general, the Mark II aerial source did a reasonable job of providing response tracking information for the array sensors, although the uniformity in the data is not as good as that shown in Figs. 16 and 17 for the A.P. Hill aerial source calibrations. There is more variability in the tracking response in particular in Fig. 21 for those microphones located in the middle and outer portions of the array aperture. This is not necessarily surprising given the large solid angle subtended by the array (39.3 degrees) with the ACV at an altitude of 350 feet. The propagation distances to the outer microphones are significantly longer than those for sensors near the center of the array, requiring larger corrections for directivity and atmospheric absorption. Any additional factors influencing the sound propagation beyond those assumed in the corrections will diminish the effectiveness of those corrections.

One effect that has not been investigated in detail to date is the ACV rotor downwash affecting the propagation of sound through the ensuing shear layer under the vehicle. The Mark I ACV was a smaller, lighter vehicle (see Table I), and thus exhibited less thrust from individual rotors with a corresponding decrease in the strength of the downwash. Coupled with the much smaller array aperture utilized at Fort A.P. Hill (where the microphones were more centrally located under the vehicle) this could account for the better tracking performance shown in Figs. 16 and 17. Some evidence for the effect of array size coupled with ACV rotor downwash can be seen in Figure 22 where two ground footprint noise contours for representative Mark I and Mark II ACV runs are shown. These footprints are obtained via a linear interpolation of the levels recorded by the microphones at a frequency of $4 \mathrm{kHz}$, projected onto a uniformlyspaced Cartesian grid. For reference, the black circle in Fig. 22b denotes the perimeter of the A.P. Hill array aperture superimposed on the much larger aperture used at Edwards. The uniformity of the footprint for the smaller array aperture is clearly shown in Fig. 22a. This is contrasted with the contours shown in Fig. $22 \mathrm{~b}$ where partial uniformity is observed only near the center of the array. More work is needed to understand the full effects of the vehicle aerodynamics on the fidelity of measuring the sound generated by the aerial sound source. Nonetheless, as a result of the current observations, modifications are being performed to the Mark II ACV to reduce the weight of the vehicle and thus reduce the rotor downwash strength. One of the lessons learned from the Edwards AFB deployment is that considerations of ACV vehicle weight, number of rotors, and the location of the sound source in relation to the downwash are factors that should be considered in minimizing the variability in microphone tracking responses.

Comparison of Methods: Figure 23 shows a comparison of tracking performance between the aerial and ground source methods conducted at Edwards. Four representative microphones are shown taken individually from the central plate and the inner, middle, and outer regions of the array aperture. For clarity, the $95 \%$ confidence intervals are only shown for the aerial source data; however, the error bars are of a similar size for the ground source data. It is immediately apparent from an examination of the data that almost perfect agreement in the tracking is observed for the microphone on the array central plate. As one moves to microphones farther away from the center of the array, the agreement between the two calibration methods degrades but is still quite good for the majority of the runs shown when taking into account the overlap in the $95 \%$ confidence intervals for the data. There are some periods, for instance in runs 6 through 9 in Fig. 23c, where significant deviations are shown between the two methods. However, these deviations decrease (at least for this microphone) toward the end of the displayed runs. It can be concluded from this comparison of methods that no one calibration technique is perfect when working under the sometimes harsh ambient conditions that field-deployable arrays and calibration systems are exposed. Therefore, the use of multiple response tracking methods like the two described herein is justified in order to allow better decisions to be made during a deployment regarding the health of the individual array sensors.

17 of 22

American Institute of Aeronautics and Astronautics 


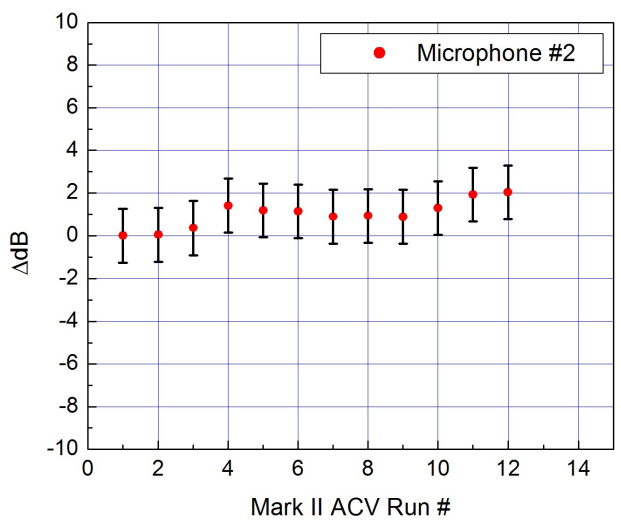

(a)

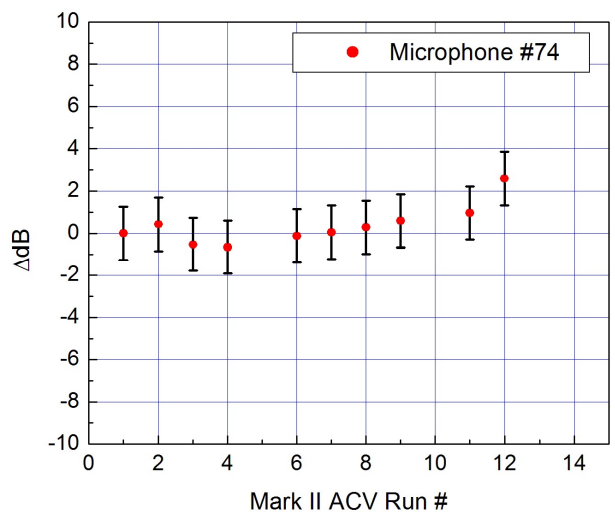

(e)

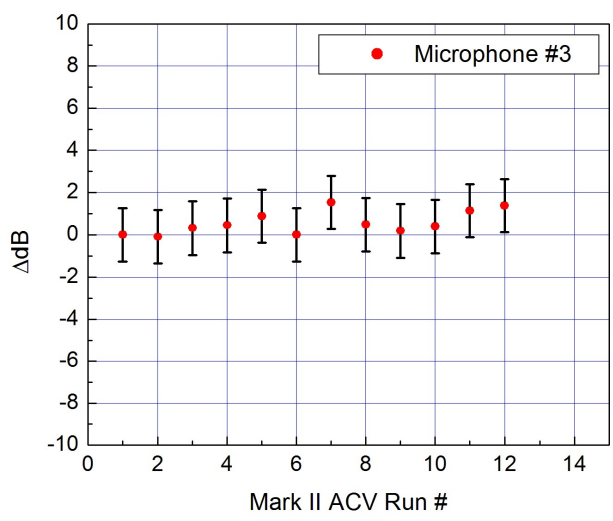

(b)

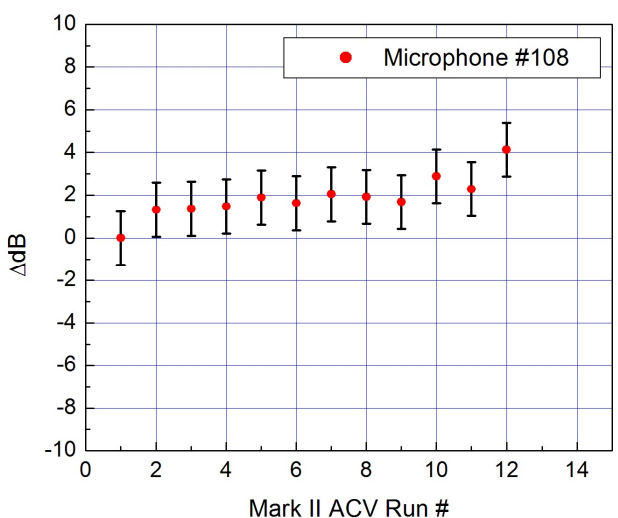

(f)

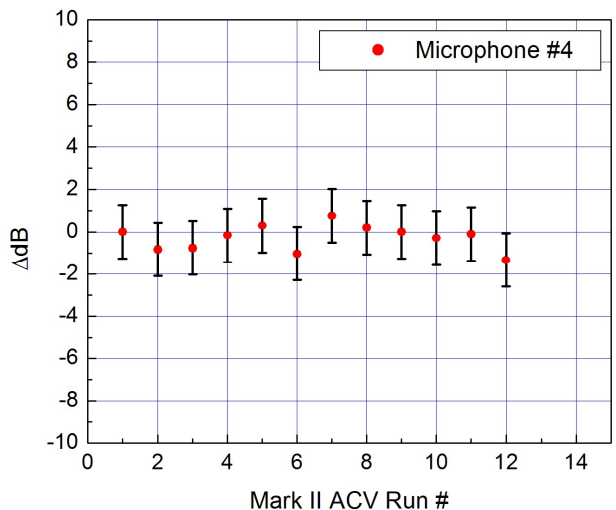

(c)

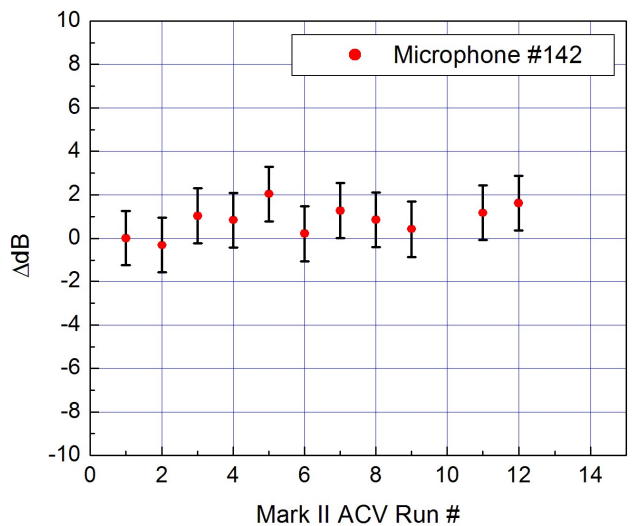

(g)
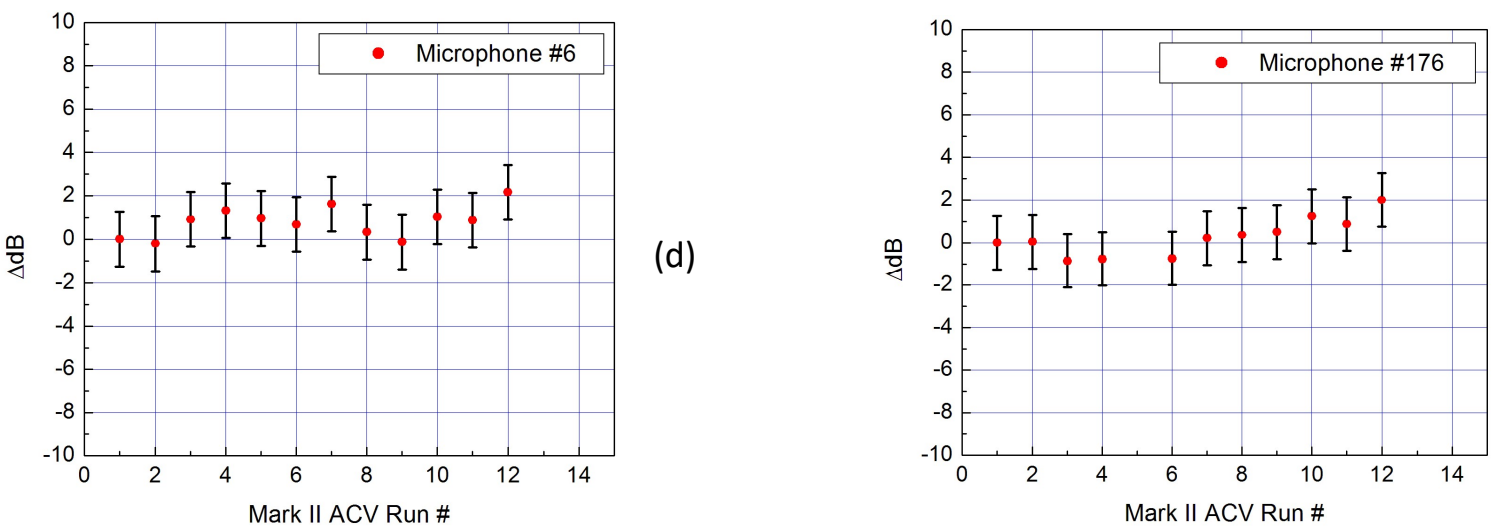

(h)

Figure 20. Tracking of measured microphone levels for 12 separate Mark II ACV runs conducted at Edwards AFB from August 18 - October 5, 2016.

(a) - (d) are microphones on central plate, (e) - (h) are individual inner microphones 


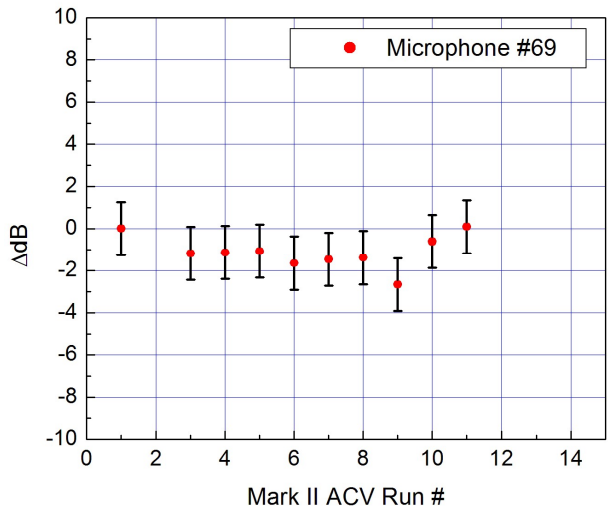

(a)

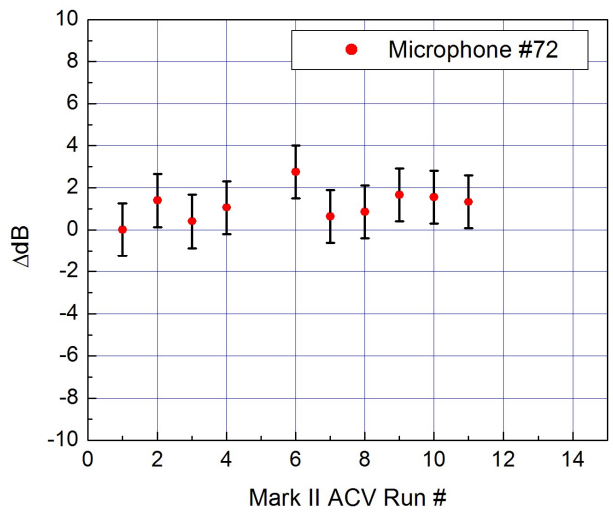

(e)

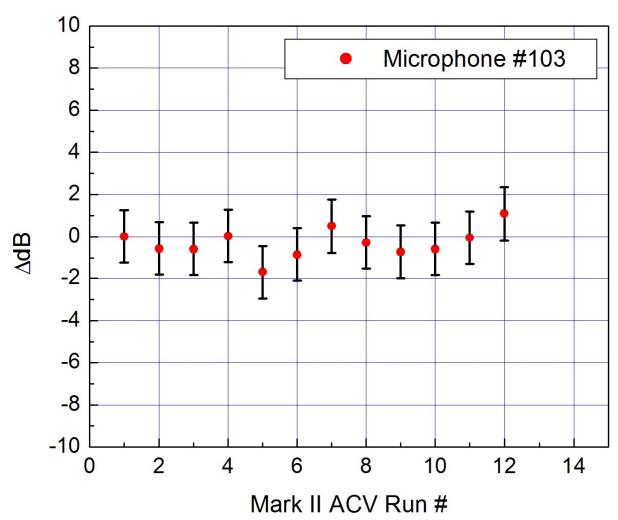

(b)

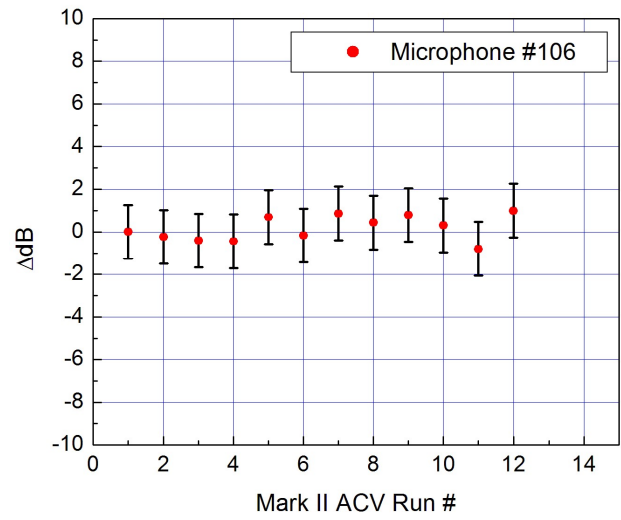

(f)

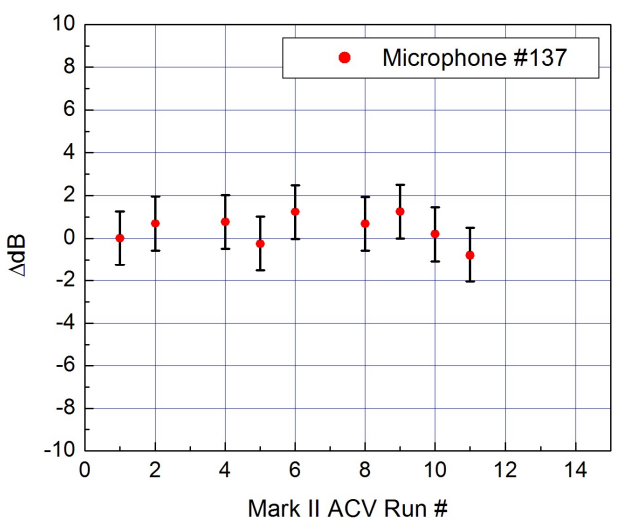

(c)

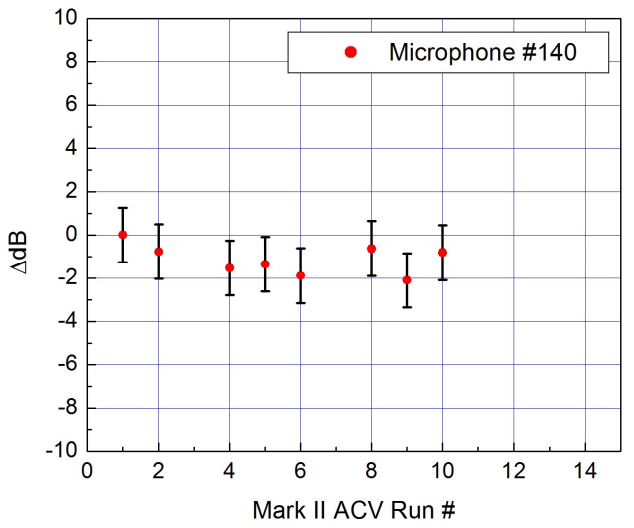

(g)
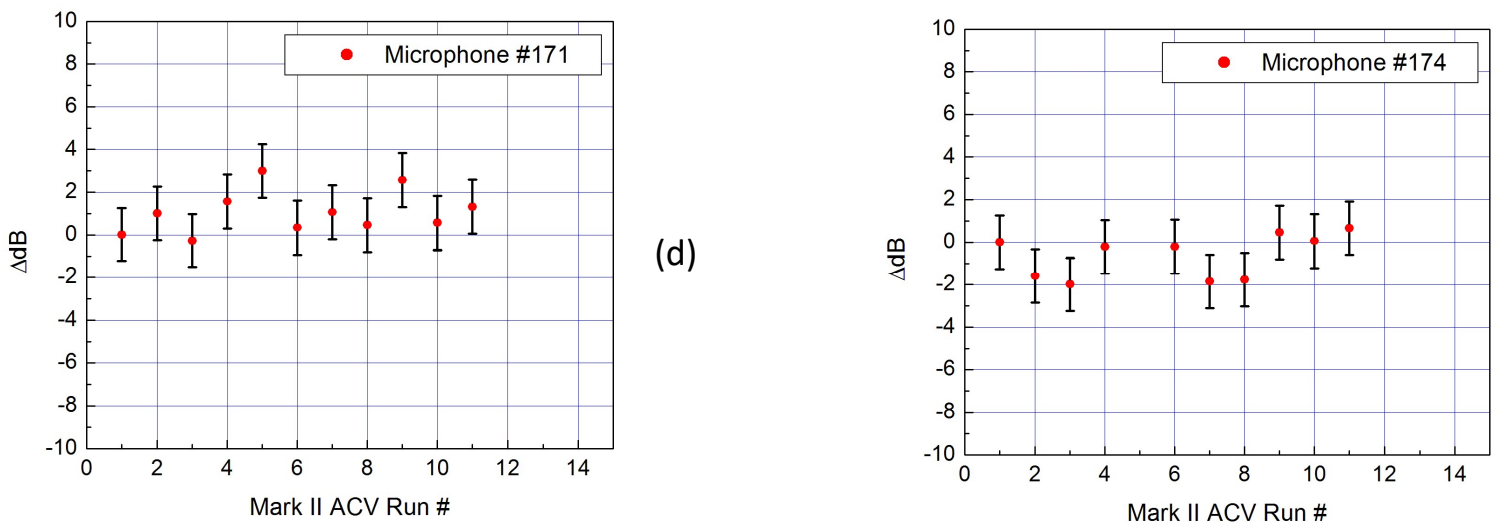

(h)

Figure 21. Tracking of measured microphone levels for 12 separate Mark II ACV runs conducted at Edwards AFB from August 18 - October 5, 2016.

(a) - (d) are individual middle microphones, (e) - (h) are individual outer microphones 

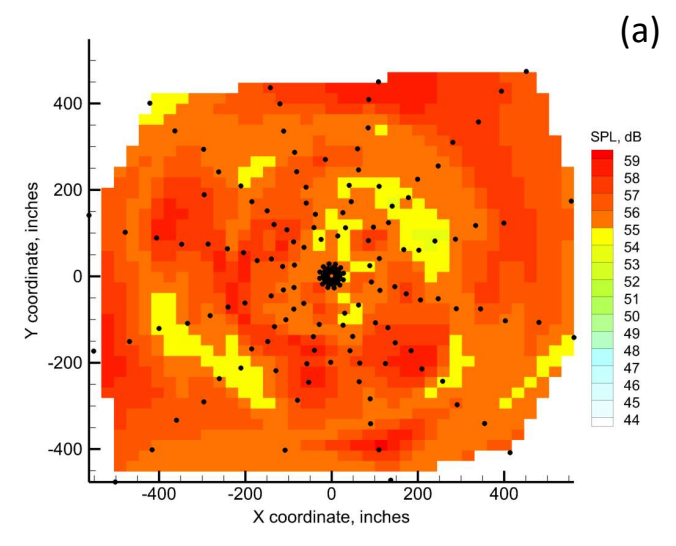

(b)

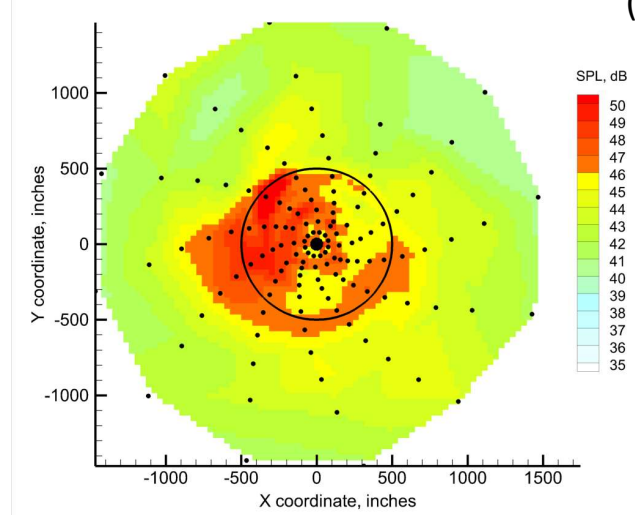

Figure 22. Representative 4-kHz noise footprints for ACV flights.

(a) Mark I ACV, Fort A.P. Hill, altitude $=400$ feet

(b) Mark II ACV, Edwards, altitude $=\mathbf{3 5 0}$ feet

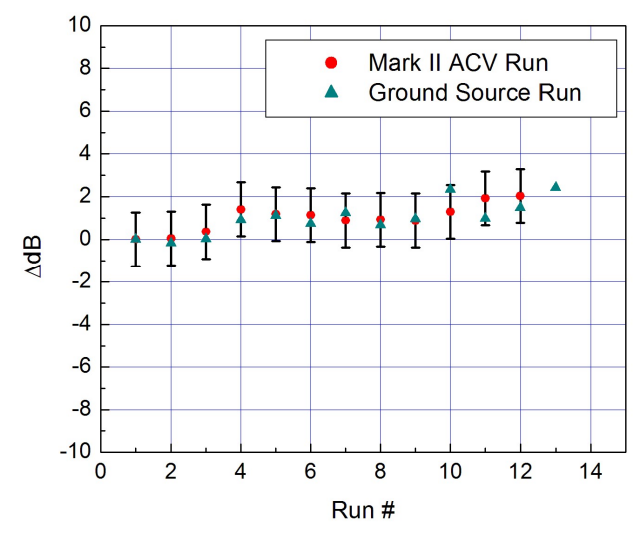

(a)

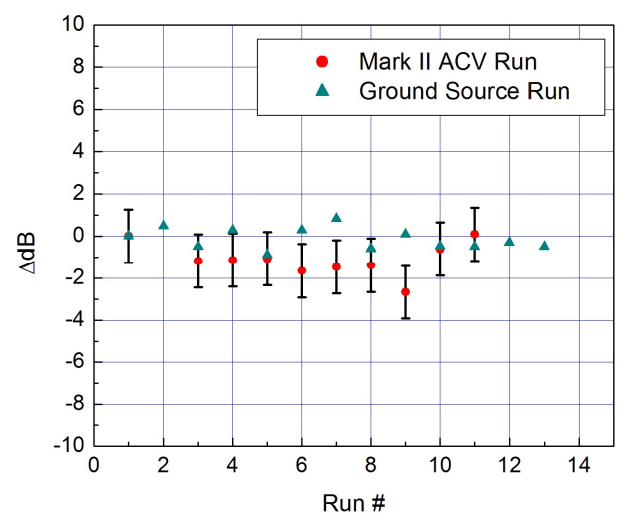

(c)
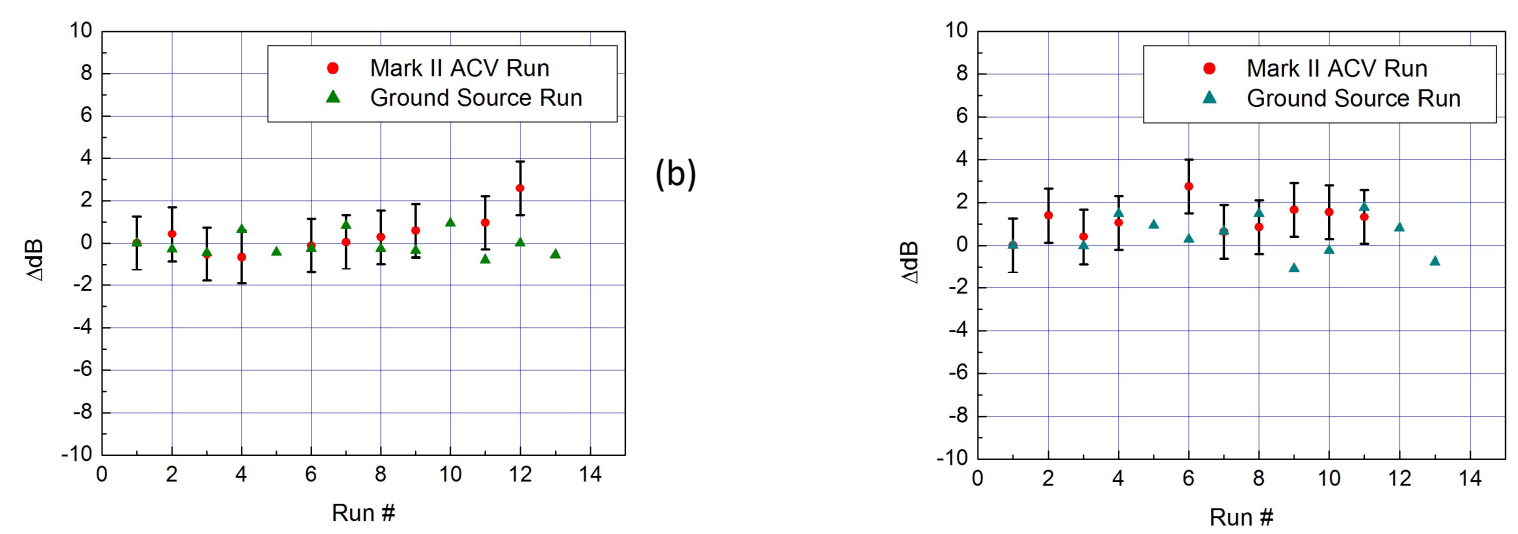

(d)

Figure 23. Comparison of tracking performance between ground source and Mark II ACV runs conducted at Edwards AFB from August 18 - October 5, 2016.

(a) central plate microphone \#2, (b) inner microphone \#74, (c) middle microphone \#69, (d) outer microphone \#72 


\section{V.Summary}

Over the past few years new aeroacoustic measurement capabilities have been developed by NASA for use in flyover testing of airframe and propulsive noise for a range of aircraft types and scales. The instrumentation consists of large channel-count, field-deployable microphone phased arrays incorporating up to 185 hardened, weather-resistant sensors. Depending on the duration of the test campaign, the microphones may be required to stay deployed at the testing site for weeks or even months. This presents a challenge in regards to tracking the response (i.e., sensitivity) of the individual sensors as a function of time in order to both estimate the uncertainty in the acoustic measurements and assess the health of the array. To address this challenge, two different methods for in-situ tracking of microphone responses have been described. The first relies on the use of an aerial sound source attached as a payload on a hovering acoustic calibration vehicle. The second relies on the use of individually excited ground-based sound sources strategically placed throughout the array pattern. Testing of the two methods was performed in microphone array deployments conducted at Fort A.P. Hill in 2015 and at Edwards Air Force Base in 2016. The results indicate that the drift in individual sensor responses can be tracked reasonably well using both methods. The aerial sound source method can be more problematic depending on the size of the array aperture. Good performance was demonstrated using this method during the A.P. Hill deployment where a restricted aperture size was employed. However, increased variability in the recorded microphone levels was observed during the Edwards deployment where a large array aperture was used. The degradation in performance, which mainly affected microphones in the middle and outer portions of the array farther from the array center, was most likely due to a combination of complex atmospheric propagation, more pronounced sound source directivity, and increased sound scatter through the rotor downwash shear layer on the larger ACV utilized at Edwards. Nevertheless, in-situ response tracking methods are useful as a diagnostic tool for monitoring the calibration of a phased array during long-duration deployments, and the use of several calibration methods is preferred during a deployment to allow valid decisions to be made regarding the health of the array sensors.

\section{Acknowledgments}

The authors gratefully acknowledge the support and expertise of the NASA Langley and NASA Armstrong UAS Operations Offices in coordinating the sUAS testing conducted at Fort A.P. Hill and Edwards AFB. The authors also gratefully acknowledge the support of personnel at the NASA Armstrong Flight Research Center in the coordination and set up of the array hardware at Edwards AFB. This work was funded by the NASA Environmentally Responsible Aviation (ERA) Project under Integrated Technology Demonstration activity (ITD) 50A and by the NASA Flight Demonstrations and Capabilities (FDC) Project.

\section{References}

${ }^{1}$ Humphreys, W. M., Jr., Brooks, T. F., Hunter, W. W., Jr., and Meadows, K. R., "Design and Use of Microphone Directional Arrays for Aeroacoustic Measurements," AIAA Paper 1998-0471, 36st Aerospace Sciences Meeting and Exhibit, Reno, NV, 1998.

${ }^{2}$ Humphreys, W. M., Jr., Brooks, T. F., Bahr, C. J., Spalt, T. B., Bartram, S. M., Culliton, W. G., and Becker, L. E., "Development of a Microphone Phased Array Capability for the Langley 14- by 22-foot Subsonic Tunnel," AIAA Paper 2014-2343, $20^{\text {th }}$ AIAA/CEAS Aeroacoustics Conference, Atlanta, GA, 2014. 
${ }^{3}$ Khorrami, M. R., Lockard, D. P., Humphreys, W. M., Jr., Choudhari, M. M., and Van de Ven, T., "Preliminary Analysis of Acoustic Measurements from the NASA-Gulfstream Airframe Noise Flight Test," AIAA Paper 2008-2814, 14 ${ }^{\text {th }}$ AIAA/CEAS Aeroacoustics Conference, Vancouver, Canada, 2008.

${ }^{4}$ Humphreys, W. M. Jr., Lockard, D. P., Khorrami, M. R., Culliton, W. G., McSwain, R. G., Ravetta, P. A., and Johns, Z., "Development and Calibration of a Field-Deployable Microphone Phased Array for Propulsion and Airframe Noise Flyover Measurements," AIAA Paper 2016-2898, 22 ${ }^{\text {nd }}$ AIAA/CEAS Aeroacoustics Conference, Lyon, France, 2016.

${ }^{5}$ True, H. C., "The Layered Weather Correction for Flyover Noise Testing," AIAA Paper 1976-0895, AIAA Aircraft Systems and Technology Meeting, Dallas, TX, 1976.

${ }^{6}$ ANSI Standard, "Method for the Calculation of the Absorption of Sound by the Atmosphere," ANSI S1.26-1978, American National Standards Institute, Inc., June 23, 1978.

${ }^{7}$ Eargle, J., Loudspeaker Handbook, $2^{\text {nd }}$ Edition, Kluwer Academic Publishers, 2003.

${ }^{8}$ Welch, P.D., "The Use of Fast Fourier Transform for the Estimation of Power Spectra: A Method Based on Time Averaging Over Short Modified Periodograms," IEEE Transactions on Audio and Electroacoustics, Vol. AU-15, pp. 70-73, 1967.

${ }^{9}$ Dieck, R.H., Measurement Uncertainty, $4^{\text {th }}$ Edition, ISA Publishers, 2007. 